\title{
LES MASSES DE SÉDIMENTS MARQUÉS A INJECTER DANS UNE EXPÉRIENCE DE TRACEURS RADIOACTIFS EN SÉDIMENTOLOGIE DYNAMIQUE
}

\author{
par G. Courtois * \\ ef G. Sauzay **
}

\section{Résumé}

Lorsqu'un détecteur se trouve en présence d'une activité répartie en plusieurs entités radioactives distinctes, le taux de comptage qu'il fournit est fonction du nombre d'entités et de leur répartition dans l'espace entourant le détecteur. Les auteurs se proposent de donner une théorie générale de la variation de réponse du détecteur, et de la probabilité associée à chaque valeur de cette réponse, quand varient à activité constante, le nombre et la disposition des entités dans un volume donné

Ils appliquent la théorie ainsi développée au problème de la détection d'un nuage de traceurs dans les expériences de sédimentologie, menées au moyen de traceurs radioactifs.

Dans un premier temps, le calcul est fait avec un traceur donné $\left({ }^{192} \mathrm{I} r\right)$ réparti à la surface du lit, pour un sable de granulométrie uniforme, pour un détecteur donné de réponse géométrique connue; les auteurs étudient d'abord une détection statique du nuage (c'est-à-dire faite point par point) puis une détection dynamique (sonde traînée sur le lit). Ils obtiennent dans ces deux cas la masse minimale de traceur par unité d'activité qu'il est nécessaire d'immerger afin d'obtenir en limite du nuage radioactif, une fluctuation du taux de comptage due à la fluctuation de position des grains radioactifs, égale à $30 \%$ du taux de comptage moyen.

Puis les auteurs cherchent à généraliser les résultats précédents pour pouvoir déterminer cette masse minimale, quand on utilise d'autres détecteurs, d'autres traceurs, d'autres granulométries, en tenant compte du mode de décodage de l'information (numérique ou analogique) et de l'enfouissement du traceur.

Ils obtiennent ainsi des formules générales tenant compte des paramètres précédents et permettant de déterminer les quantités de sable à immerger en fonction des paramètres de mesure et des caractéristiques du sédiment.

Pour terminer, on examine le cas particulier de la prise des carottes.

Les auteurs montrent qu'une détection dynamique, technique qu'ils préconisent, nécessite une masse de traceurs par unité d'activité dix fois plus faible qu'une détection statique, celle-ci à son tour demandant dix fois moins de traceurs qu'une étude avec des carottages.

\section{1. - Position du problème}

\footnotetext{
; Section d'Application des Radioéléments, C.E.A., Saclay.

$\because$ Section d'Application des Radioéléments, C.E.A., Saclay. Actuellement: Section d'Hydrologie, Agence Internationale de l'Energie Atomique, Vienne (Autriche).
}

Lorsque l'on dresse un bilan bibliographique des masses de sédiments immergées dans une expérience de traceurs radioactifs, on s'aperçoit que pour des expériences assez analogues, ces masses varient dans des proportions considérables : entre $35 \mathrm{~g}$ pour certaines expériences françaises et $4 \mathrm{t}$ pour des expériences portugaises, soit dans un rapport de 1 à 100000 .

Il n'est trouvé nulle part dans la littérature de considération précise et scientifique sur le choix de ces masses qui semble laissé au hasard des goûts et des habitudes. Nous avons voulu combler cette lacune, expliquer notre point de vue et donner des informations aussi rigoureuses que possible sur le problème essentiel, maintes fois évoqué, mais jamais résolu, de la statistique du nombre de grains.

Quant à nous, nous partons du principe que nous immergeons la masse minimale de sédiment marqué, compatible avec les impératifs de l'expérience, et ne voyons aucune raison valable de dépasser cette valeur. A nos yeux, cette prise de position se justifie de la manière suivante :

1* Technologiquement, la préparation, la manipulation et le transport, l'immersion d'une faible masse de sédiment marqué sont beaucoup plus aisés que les opérations analogues pour des masses importantes. Cet aspect n'est pas le moindre et nous apparaît primordial, car nous avons basé tout notre équipement sur des ensembles légers, extrêmement maniables, qui confèrent aux expériences facilité et rapidité de mise en œuvre, associé à un bas prix de revient.

$2^{\circ}$ Du point de vue de l'hydraulique et de la dynamique des sédiments, l'idéal serait de pouvoir, lors de l'immersion, enfouir le sédiment marqué sur toute l'épaisseur de la couche en mouvement. Ceci étant impossible, l'immersion est généralement faite en surface. Cependant il importe, ce faisant, de ne pas modifier la dynamique locale et surtout, si possible, de déposer cette masse sur le fond, de telle sorte que les conditions de bon mélange, ou disons la représentativité du traceur intervienne au plus tôt et sur une surface ensemencée la plus faible.

A cet égard, nous considérons que le fait de créer sur le fond un monticule par l'immersion d'une masse importante de traceur est loin d'être favorable à l'obtention des conditions précédentes et que l'idéal serait de pourvoir ensemencer le fond du lit d'une «monocouche » de grains 


\section{G. COURTOIs et al.}

actifs pratiquement assimilés et soumis immédiatement après l'immersion aux mêmes conditions hydrauliques que les grains superficiels.

Par là même, nous sommes surpris par l'immersion de masses importantes de sédiment telle qu'elle est pratiquée systématiquement par exemple en Allemagne Fédérale et ne comprenons pas les raisons d'un mélange préalable d'une masse faible de sédiment marqué à une masse importante de sédiment inactif, tel qu'il est exercé en Grande-Bretagne.

Les considérations précédentes nous conduisent à immerger la masse minimale de traceur radioactif. L'activité étant choisie, cette masse minimale peut être déterminée par des considérations relatives à la statistique du nombre de grains actifs présents sous le détecteur aux limites de nuage radioactif. En effet, il importe que sur les bords de ce nuage, en des lieux où le signal net dû à la radioactivité artificielle reste encore significatif, il y ait sous la sonde un nombre minimal de grains actifs afin que le signal enregistré avoisine le signal moyen et fluctue dans des limites raisonnables qui permettent encore une exploitation des informations reçues.

Dans le présent article nous allons dans un premier chapitre développer des considérations qui permettent d'atteindre les lois de probabilités des répartitions des signaux reçus en fonction du nombre de grains présents sous le détecteur; ces considérations seront ensuite appliquées au cas de la sonde détectrice que nous utilisons le plus fréquemment associée à un traceur fréquent $1^{192} \mathrm{Ir}$. Enfin nous ferons la généralisation des résultats obtenus à d'autres conditions d'utilisation : traceurs divers, sondes détectrices et géométries variées, grains de traceur enfouis, etc.

Pour ne pas alourdir hors limite le présent texte, certains développements mathématiques ont été volontairement omis et on conseille alors de se reporter au texte de la thèse de Sauzay [1].

\section{2. - Principe des calculs. Caractéristiques des lois de distribution}

\subsection{Les lois de distribution des taux de comptages et leurs caractéristiques.}

Sans faire aucune hypothèse sur les conditions opératoires, supposons, lors d'une détection, la présence à un instant donné dans la «sphère d'influence » du détecteur (*) de $n$ grains radioactifs fournissant un taux de comptage net de $\mathrm{N}_{n}$

Dans ce qui va suivre, nous ne tiendrons pas compte de la fluctuation statistique des taux de comptage due au caractère aléatoire des phénomènes de désintégration radioactive.

$\mathrm{N}_{n}$ est éminemment variable avec la position des grains par rapport à la sonde, et en définitive, en envisageant les différentes possibilités d'arrangement de ces $n$ grains et la probabilité associée à chaque arrangement, il est observé une loi de densité de probabilité d'obtention du taux de comptage $\mathrm{N}_{n}$ :

$$
\Pi_{n}=\frac{d \mathrm{P}\left(\mathrm{N}_{n}\right)}{d\left(\mathrm{~N}_{n}\right)}
$$

(") La « sphère d'influence » d'un détecteur est la limite des points qui dans une géométrie défnie : milieu infini, milieu semi infini, milieu superficiel, doment $98 \%$ de l'information totale.

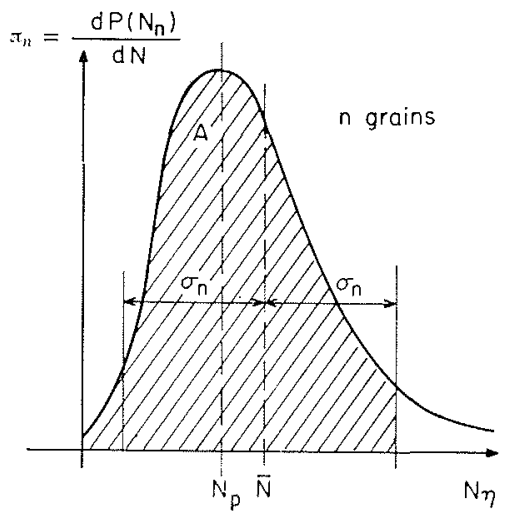

1/ Probabilité d'obtenir un taux de comptage $\mathrm{N} n$ pour $n$ grains.

La connaissance d'une telle loi (fig. 1) peut permettre de juger, à partir de certains critères, si en définitive le nombre de grains $n$ sous le détecteur peut être considéré comme suffisant pour donner une information représentative.

Nous nous proposons de déterminer les fonctions $\Pi_{n}$ en fonction de $n$ et pour chaque valeur de $n$ de caractériser cette fonction par les paramètres suivants (fig. 1).

1. Le taux de comptage le plus probable $N_{1}$, et son rapport au taux de comptage moyen $\bar{N}$ :

$$
\frac{\mathrm{N}_{\mathrm{P}}}{\overline{\mathrm{N}}}=f(n)
$$

2. L'écart quadratique de la loi:

$$
\begin{gathered}
\sigma_{n}{ }^{2}=\int\left(\mathrm{N}_{n}-\overline{\mathrm{N}}\right)^{2} \times \Pi(n) \times d \mathrm{~N}_{n} \\
\sigma_{n}=\varphi(n)
\end{gathered}
$$

3. La probabilité $A$ associée à l'écart quadratique (*).

$$
\mathrm{A}_{\sigma}=\mathrm{A}(n, \sigma)
$$

ou encore le pourcentage de cas où l'information recueillie appartient à un intervalle $\pm \Delta$ autour de la valeur moyenne $\bar{N}_{n}$ :

$$
\mathrm{A}_{\Delta}=\mathrm{A}(n, \Delta)
$$

\subsection{Calcul des distributions $\Pi_{n}$.}

Cherchons à déterminer la loi de répartition $\Pi_{n}$. Chacun des $n$ grains présents donne un taux de comptage $\mathrm{N}_{i}, i$ étant un repère individuel de chaque grain, avec:

$$
\mathrm{N}_{n}=\Sigma_{i} \mathrm{~N}_{i}
$$

Dissocions les $n$ grains en deux lots composés respectivement de $j$ et $k$ grains; l'équation (2) devient:

avec $\jmath+k=n$.

$$
\begin{aligned}
\mathrm{N}_{n} & =\sum_{i=1}^{i=j} \mathrm{~N}_{i}+\sum_{i=j+1}^{i=n} \mathrm{~N}_{i} \\
& =\mathrm{N}_{j}+\mathrm{N}_{k}
\end{aligned}
$$

D'après le principe des probabilités conditionnelles, on a : avec $\mathrm{N}_{l}=\mathrm{N}_{n}-\mathrm{N}_{n} / \mathrm{N}_{j}$

(*) On rappelle que la probabilité associée à un écart quadratique $-\sigma$ n'est égale à $68 \%$ que pour une distribution gaussienne. Dans le cas général, cette probabilité reste inconmue. 
$d \mathrm{P}\left(\mathrm{N}_{n} / \mathrm{N}_{j}\right)$ représentant la probabilité d'obtenir $\mathrm{N}_{n}$ dans l'intervalle $\mathrm{N}_{n}+d \mathrm{~N}_{n}$ sachant que $\mathrm{N}_{j}$ appartient à l'intervalle $\mathrm{N}_{j}+d \mathrm{~N}_{j}$.

La probabilité d'obtenir $N_{n}$ dans ledit intervalle, quel que soit $\mathrm{N}_{j}$, est la somme des valeurs de $d \mathrm{P}\left(\mathrm{N}_{n} / \mathrm{N}_{j}\right)$ pour toutes les valeurs de $\mathrm{N}_{j}$.

Or:

$$
d \mathrm{P}\left(\mathrm{N}_{j}\right)=\Pi_{j} d \mathrm{~N}_{j} \quad \text { et } \quad d \mathrm{P}\left(\mathrm{N}_{k}\right)=\Pi_{k} d \mathrm{~N}_{k}
$$

d'où en définitive :

$$
\Pi_{n}=\frac{d \mathrm{P}\left(\mathrm{N}_{n}\right)}{d\left(\mathrm{~N}_{n}\right)}=\int \Pi_{\mathrm{N}_{j}} \Pi_{j}\left(\mathrm{~N}_{j}\right) \times \Pi_{k}\left(\mathrm{~N}_{n}-\mathrm{N}_{j}\right) d \mathrm{~N}_{j}
$$

avec $j+k=n$.

Cette équation est absolument générale et est parfaitement indépendante du mode de détection adopté.

Supposons connue la loi de distribution $\mathrm{II}_{1}$ pour un grain. La formule (3) suggère de prendre pour valeurs de $n$ successives :

On a alors :

$$
n=1, n=2, n=4 \ldots, n=2^{\mathrm{P}}
$$

$$
\begin{aligned}
& n=1 \quad \Pi_{1} \text { connu par hypothèse } \\
& n=2 \quad j=1, \quad k=1 \\
& \Pi_{2}=\int_{N_{j}} \Pi_{1}\left(N_{j}\right) \times \Pi_{1}\left(N_{n}-N_{j}\right) d N_{j} \\
& n=4 \quad j=2, \quad k=2 \\
& \Pi_{1}=\int_{\mathrm{N}_{j}} \Pi_{2}\left(\mathrm{~N}_{j}\right) \times \Pi_{2}\left(\mathrm{~N}_{n}-\mathrm{N}_{j}\right) d \mathrm{~N}_{j} \\
& n=2^{\mathrm{p}} \quad j=2^{\mathrm{p}-1}, \quad k=2^{\mathrm{p}-\mathrm{1}} \\
& \Pi_{2_{p}}=\iint_{x_{j}} \Pi_{2_{p-1}}\left(\mathrm{~N}_{j}\right) \times \Pi_{2_{p-1}}\left(\mathrm{~N}_{n}-\mathrm{N}_{j}\right) d \mathrm{~N}_{j}
\end{aligned}
$$

On pourra ainsi par récurrence déterminer les fonctions $I_{\left.2^{2}\right]}$ successives, puisque dans chaque calcul intervient uniquement la fonction $\Pi_{2_{p-1}-1}$ déterminée au calcul précédent.

\section{NOTES :}

1. Si nécessaire, on peut obtenir $\Pi_{n}$ pour une valeur quelconque de $n$ en combinant les fonctions $\Pi_{2_{i j}}$.

Par exemple :

$-n=33$ obtenu avec $j=32, k=1$ :

$$
\Pi_{33}=\int_{\mathrm{N}_{3}} \Pi_{32}\left(\mathrm{~N}_{j}\right) \times \Pi_{1}\left(\mathrm{~N}_{n}-\mathrm{N}_{j}\right) d \mathrm{~N}_{j}
$$

$-n=25$ obtenu:

$$
\text { en déterminant } \Pi_{24} \text { avec } j=16 \text { et } k=8
$$$$
\text { puis } \Pi_{25} \text { avec } j=24 \text { et } k=1
$$

2. La seule connaissance de $\mathrm{II}_{1}$ entrâne la connaissance de tout $\Pi_{n}$ indépendamment de toute hypothèse. Nous reprendrons ce point un peu plus loin en insistant sur ces conséquences.

\subsection{Calcul des écarts quadratiques moyens. Théorème cen-} tral limite.

L'application đu théorème central limite [2] au problème envisagé montre que la densité de probabilité $\Pi_{n}$ tend vers une loi de Gauss quand $n$ augmente infiniment, et ceci quelle que soit la loi $\Pi_{1}$ observée.
De plus l'application de ce théorème au problème envisagé, donne une relation entre les écarts quadratiques successifs :

$$
\sigma_{n}=n^{1 / 2} \sigma_{1}
$$

Ceci signifie qu'en prenant pour variable réduite:

$$
\mathrm{Z}=\frac{\mathrm{N}_{n}-\overline{\mathrm{N}}_{n}}{\sigma_{n}}=\frac{\sum_{n} \mathrm{~N}_{i}-n \mathrm{~N}_{1}}{n^{1 / 2} \cdot \sigma_{1}}
$$

La loi de probabilité $d P(z) / d z$ tend vers la loi de Gauss, avec :

$$
\mathrm{P}(z)=\frac{1}{(2 \Pi)^{1 / 2}} \int_{-\infty}^{z} e^{-z / 2 / 2} d \mathrm{Z}
$$

En pratique, dans ce qui va suivre, on augmentera le nombre de grains $n$ par $\mathrm{m}^{2}$ mais pour une activité totale constante afin d'obtenir toujours le même taux de comptage moyen:

$$
\overline{\mathrm{N}}_{1}=\overline{\mathrm{N}}_{n}=\overline{\mathrm{N}}
$$

Ceci revient à présenter la variable réduite sous la forme :

$$
\mathrm{Z}=\frac{(1 / n)\left(\mathrm{N}_{n}-\overline{\mathrm{N}}_{n}\right)}{(1 / n) \sigma_{n}}=\frac{\left.(1 / n) \Sigma_{n} \mathrm{~N}_{i}-\overline{\mathrm{N}}\right)}{\sigma_{1} / \sqrt{n}}
$$

Avec par suite :

$$
\sigma_{n}=n^{-1 / 2} \sigma_{1}
$$

Ainsi par l'établissement de la formule ci-dessus, le théorème central limite permet immédiatement de connaitre l'écart quadratique correspondant à la «vision 》 de $n$ grains par la sonde, si on connaît l'écart dû à 1 grain; on voit que plus le nombre de grains sera grand, moins la distribution des taux de comptage sera étalée.

Notons que $\sigma_{1}$ peut être calculé par application du théorème de Koenig [2]:

$$
\sigma_{1}^{2}+\bar{N}^{2}=\int_{N_{1}} N_{1}^{2} \times \Pi_{1}\left(N_{1}\right) d N_{1}
$$

puisque l'on suppose connu $\Pi_{1}$.

Cependant, le théorème central limite ne permet pas d'associer une probabilité à l'écart trouvé $\sigma_{n}$; on sait seulement que si $n$ croit indéfiniment, la loi des distributions de $\mathrm{N}_{n}$ tendant vers une loi de Gauss, la probabilité associée à $\sigma_{n}$ tend vers $68 \%$ selon $\mathrm{N}_{n}=\overline{\mathrm{N}} \pm \sigma_{n}$ à $68 \%$ de degré de confiance.

\subsection{Probabilités associées aux écarts quadrakiques.}

Ayant déterminé la loi $\Pi_{n}$ et son écart associé, on aura la probabilité associée en mesurant graphiquement l'aire A définie par $\pm 1 \sigma$ autour de la valeur moyenne (fig. 1).

De même, si on s'intéresse au pourcentage des cas où linformation recueillie appartient à un intervalle $\pm \Delta$ autour de la valcur moyenne, on déterminera graphiquement sur la courbe représentative de $\Pi_{n}$, la valeur de la surface limitée par les bornes de cet intervalle.

\subsection{Conclusions.}

Ainsi donc, à partir de la connaissance de la loi des probabilités des taux de comptage pour un grain unique, on a 


\section{G. CoURToIs et al.}

mis en évidence un procédé de calcul qui permet de déterminer :

- les lois de probabilités pour $n$ grains et plus particulièrement pour $2,4, \ldots, 2 p$ grains;

- les écarts quadratiques de ces lois;

- les probabilités associées à ces écarts.

Ce vrocessus mathématique est absolument général et peut-êtic appliqué à tout problème dans lequel intervient une dilui on d'entités marquées dans un milieu inactif. Il est notamment complètement indépendant des conditions expérimentales: appareillage de détection (nombre de détecteurs, nature, sensibilité), mode de détection (statique ou dynamique, information analogique ou digitale), répartition granulométrique, répartition d'activité, répartition en profondeur, géométrie et composition du milieu, énergie du rayonnement, etc.

L'essentiel est de déterminer dans les conditions réelles retenues pour la mesure, la loi de probabilité précédente pour un grain, les fonctions étudiées $f(n), \varphi(n), \mathrm{A}(n, \Delta)$ découlant alors de l'application des procédés exposés.

Nous allons maintenant examiner une telle application à quelques cas concrets, utilisant les conditions d'appareillage et de détection fréquemment rencontrées en France en sédimentologie dynamique. Puis, il sera fait une généralisation à des conditions les plus universelles possibles ou des conditions particulières de détection rencontrées en France.

\section{3. - Applications}

\subsection{Conditions expérimentales.}

L'application des considérations précédentes va être faite à des détections menées dans les conditions expérimentales suivantes.

La sonde détectrice est constituée par un unique détecteur à scintillation équipé d'un cristal INa $1^{\prime \prime} 1$ 位 $X 1^{\prime \prime}$ situé à $5 \mathrm{~cm}$ du fond et protégé par une enveloppe d'acier de $2 \mathrm{~mm}$ (fig. 2). Elle travaille en discriminateur avec un seuil réglé à $50 \mathrm{KeV}$.

Le traceur est de $1^{1} 192$ Ir et le bruit de fond est tel que nous prendrons comme significative toute information nette égale ou supérieure à $50 \mathrm{cps}$. Nous supposons de plus que, sur les bords du nuage radioactif où ce signal est obtenu, les grains sont uniquement répartis en surface et aucun d'eux n'est enfoui. Nous supposons enfin que le marquage est massique, c'est-à-dire que l'activité de chaque grain est proportionnelle à sa masse.

Dans ces conditions, les étalonnages en laboratoire montrent que :

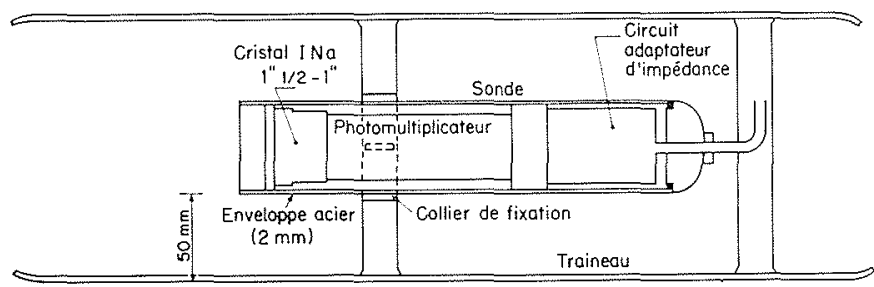

2/ Géométrie de détection adoptée en France.
- $98 \%$ de l'information provient d'une surface de $1 \mathrm{~m}^{2}$, la « sphère » d'influence étant, dans ce cas particulier, un cercle de diamètre $56 \mathrm{~cm}$;

- $50 \mathrm{cps}$ nets correspondent au signal fourni par $1 \mathrm{\mu Ci} / \mathrm{m}^{2}$ d'192 Ir uniformément réparti.

Ainsi qu'il a déjà été souligné, rappelons que nous ne nous intéressons qu'aux fluctuations dues à la statistique du nombre de grains indépendamment de celles dues à la statistique des taux de comptage, liée au caractère aléatoire des césintégrations.

\subsection{Détection statique.}

\subsubsection{Définition de LA DÉtection STATIQUe.}

On appelle détection statique, une détection dans laquelle la sonde est immobile sur le fond à examiner, pendant tout le temps où est recueillie l'information. Ce faisant la sonde n'observe qu'une seule disposition des grains, celle correspondant au lieu ou elle détecte.

Ce type de détection est pratiqué par les équipes australiennes et yougoslaves.

\subsubsection{DÉtection STATIQUe ET GRANUlométriE UNIFORME.}

\section{Calculs.}

Dans ce premier cas nous allons supposer tous les grains de même diamètre, donc de même activité, et faire crôtre le nombre de grains à activité totale constante de $1 \mu \mathrm{Ci}$ jusqu'à ce que la statistique nous semble satisfaisante.

Pour les conditions définies en 3.1 un étalonnage en laboratoire donne le nombre de chocs par seconde, fournis par 1 grain de $1 \mathrm{\mu Ci}$ d'192 $\mathrm{Ir}$, situé à une distance $r$ de l'axe du cristal: $\mathrm{N}_{1}(r)$ (fig. 3).

La probabilité élémentaire qu'un grain soit à une distance $r, r+d r$ du détecteur est :

$$
d \mathrm{P}=2 \Pi r d r
$$

La loi de probabilité $\Pi_{1}$ est donc:

$$
\Pi_{\mathrm{J}}=\frac{d \mathrm{P}}{d \mathrm{~N}_{1}}=2 \Pi r \frac{d r}{d \mathrm{~N}_{1}}
$$

$d r / d \mathrm{~N}_{1}$ étant obtenu à partir de la courbe de la figure 3 , la fonction $\Pi_{1}$ peut être construite point par point et est représentée par la courbe de la figure 4.

Cette courbe, droite en coordonnées log-log, peut être décrite par la fonction :

$$
\Pi_{1}\left(N_{1}\right)=0,431 \mathrm{~N}_{1}^{-1,3 i}
$$

pour un grain d'activité $a_{1}=1 \mu \mathrm{Ci}$ avec :

$$
\begin{gathered}
\mathrm{N}_{1 \min }=1,2 \mathrm{cps}<\mathrm{N}_{1}<920 \mathrm{cps}=\mathrm{N}_{1 \max } \\
\mathrm{N}_{\mathrm{p}}=1,2 \mathrm{cps} ; \overline{\mathrm{N}}=50 \mathrm{cps}
\end{gathered}
$$

et l'écart quadratique obtenu par le théorème de Koenig $\sigma_{1}=125 \mathrm{cps}$.

Pour deux grains d'activité unitaire $0,5 \mu \mathrm{Ci}$, nous aurons :

$$
\Pi_{2}=\int_{N_{1}} \Pi_{1}\left(N_{1}\right) \times \Pi_{1}\left(N_{2}-N_{1}\right) d N_{2}
$$

avec :

$$
\Pi_{1}=0,215 \mathrm{~N}_{1}-1,3 \pi
$$

0,215 pour tenir compte de l'activité moitié de chaque grain $(2$ grains $=$ total $1 \mu \mathrm{Ci})$. 


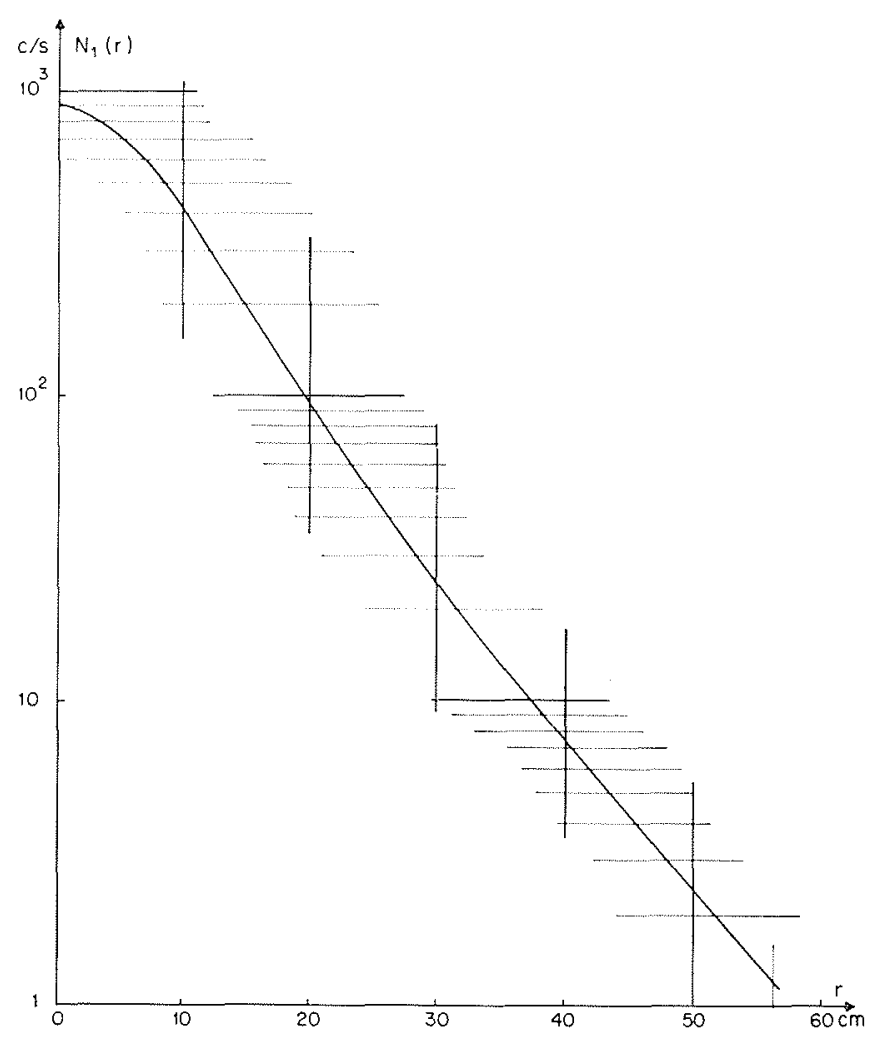

3/ Coube de réponse de l'ensemble de détection à un grain de $1, \mathrm{Ci}$ à une distance $r$ de cet ensemble.

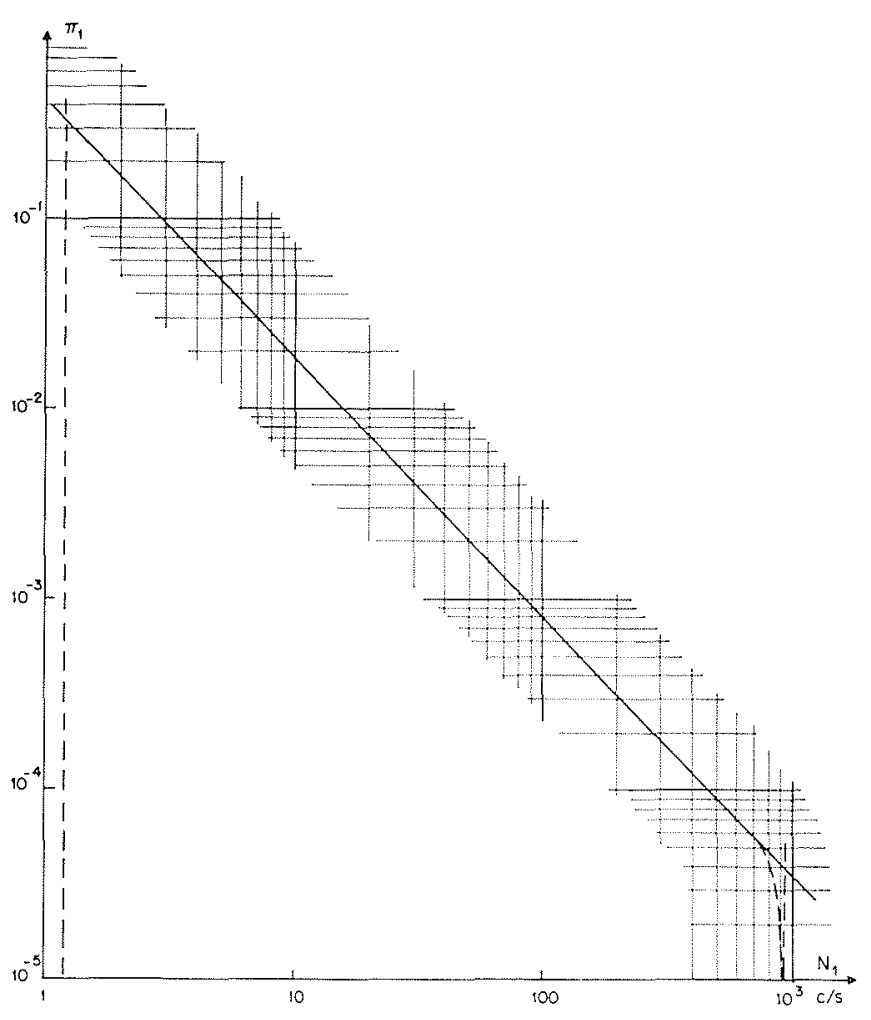

4/ Détection statique. Densité de probabilité $\mathrm{II}_{1}(\mathrm{~N})$.
En faisant fort attention aux bornes de cette intégrale qui sont fonction de la position de $N_{2}$ par rapport à $\mathrm{N}_{1 \max }$ et $N_{1 \text { min }}$, les intégrales peuvent être calculées soit graphiquement, soit mieux par l'intermédiaire d'un calculateur [voir note en fin d'article]. Le programme est chargé de calculer la fonction de probabilité $\Pi_{2}$, son écart quadratique $\sigma_{2 .}$ el à titre de vérification la valeur moyenne:

$$
\overline{\mathrm{N}}=-\overline{\mathrm{N}}_{1}=\overline{\mathrm{N}}_{2}=50 \mathrm{cps}
$$

On trouve alors:

l'écart quadratique :

$$
\sigma_{2}=88,4 \mathrm{cps}
$$

la valeur la plus probable:

$$
\left[\mathrm{N}_{\mathrm{P}}\right]_{2}=3,5 \mathrm{cps}
$$

On voit donc que pour deux grains, la valeur la plus probable est très éloignée de la valeur moyenne, et que l'écart quadratique est très grand.

Pour un nombre supérieur de grains, le processus défini au chapitre 2, combiné avec le programme précédent permet de calculer les données correspondantes à $4,8,16, \ldots$, $2^{p}$ grains.

Examinons les résultats :

FIG. 5. - Fonctions de probabilité $\Pi_{n}$ pour $n=16,32$ et 64 ;

FIG. 6. $-\mathrm{N}_{p}$ et $\mathrm{N}_{p} / \overline{\mathrm{N}}=f(n)$, relatifs aux taux de comptage le plus probable;

FIG. 7. - L'écart quadratique $\sigma_{n}$ et sa probabilité associée $\mathrm{A}(n, \sigma)$.

On vérifie à cette occasion que le $\sigma_{n}$ suit bien la loi théorique :

$$
\sigma_{n}=125 / \sqrt{n}
$$

Fig. 8. - Les pourcentages de cas possibles appartenant à un intervalle donné $N \pm \Delta$ ( $N$ et $\Delta$ en cps) :

$$
\mathrm{A}(n, \sigma) \quad \text { avec } \Delta=10, \Delta=15, \Delta=20, \Delta=40 \text {. }
$$

Masse minimale de sédiment marqué à immerger.

L'utilisation des résultats précédents est extrêmement aisée, une fois choisies les conditions aux limites de la détection, conditions qui peuvent être plus ou moins sévères suivant les utilisateurs.

Quant à nous, considérant d'une part que l'immense majorité des informations est en provenance de zones à taux de comptage beaucoup plus élevés, d'autre part que la répartition en surface des grains conduit à la déviation standard la plus grande (voir plus loin en 4.2), nous choisissons comme condition aux limites, un écart quadratique relatif égal à $30 \%$ de l'information moyenne.

Dans notre cas, l'information moyenne est de $50 \mathrm{cps}$ et l'écart quadratique sera donc de $15 \mathrm{cps}$, qui définit $n$ par la relation (9):

$$
\sigma_{n}=15=\frac{125}{\sqrt{n}}
$$

donne $n=69$ grains.

Pour une telle valeur, la figure 7 montre que la probabilité associée est de $68 \%$ comme pour une fonction Gaussienne.

Il faut donc 69 grains par $\mu \mathrm{Ci}$ d'192 $\mathrm{Ir}$ correspondant à $\mathrm{P}$ kilogrammes de sédiment par $\mathrm{Ci}$ :

$$
\mathrm{Pkg} / \mathrm{Ci}=96 d^{3}
$$

$d$ étant le diamètre des grains en mm.

Ainsi pour un diamètre de $1 \mathrm{~mm}$ ceci conduit à $96 \mathrm{~kg}$ par $\mathrm{Ci} \mathrm{d}^{192} \mathrm{Ir}$ et pour un diamètre de $0,1 \mathrm{~mm}$ à $96 \mathrm{~g}$ par Ci d'192Ir. 


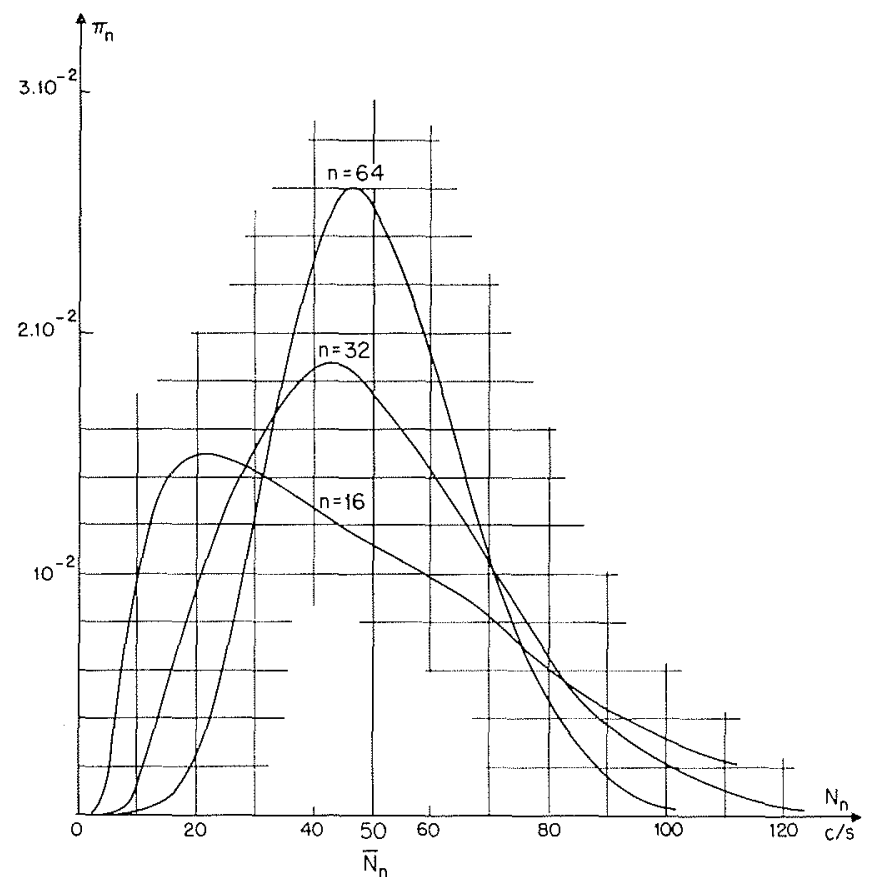

5/ Détection statique. Densité de probabilité $\Pi_{16}, \Pi_{32}, \Pi_{01}$.

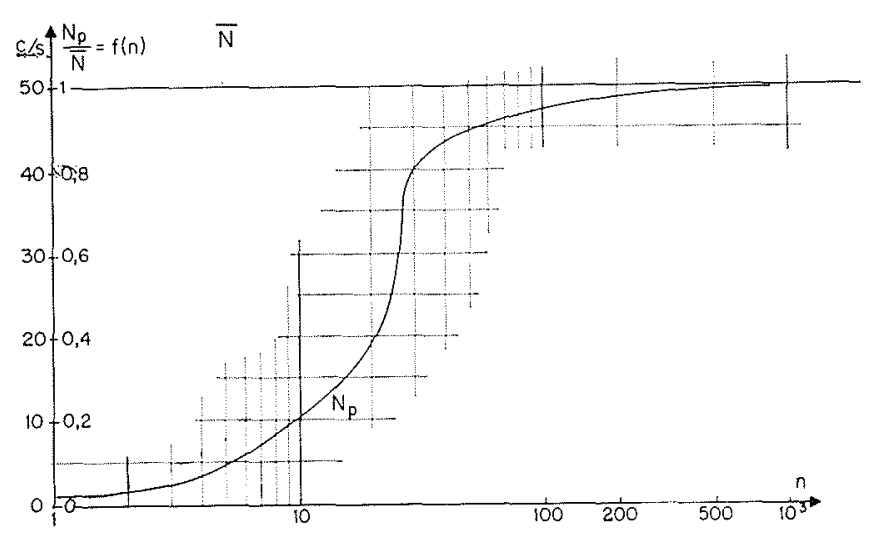

6/ Détection statique. $\mathrm{N}_{p}$ et $\mathrm{N}_{p} / \overline{\mathrm{N}}$ en fonction du nombre de grains.

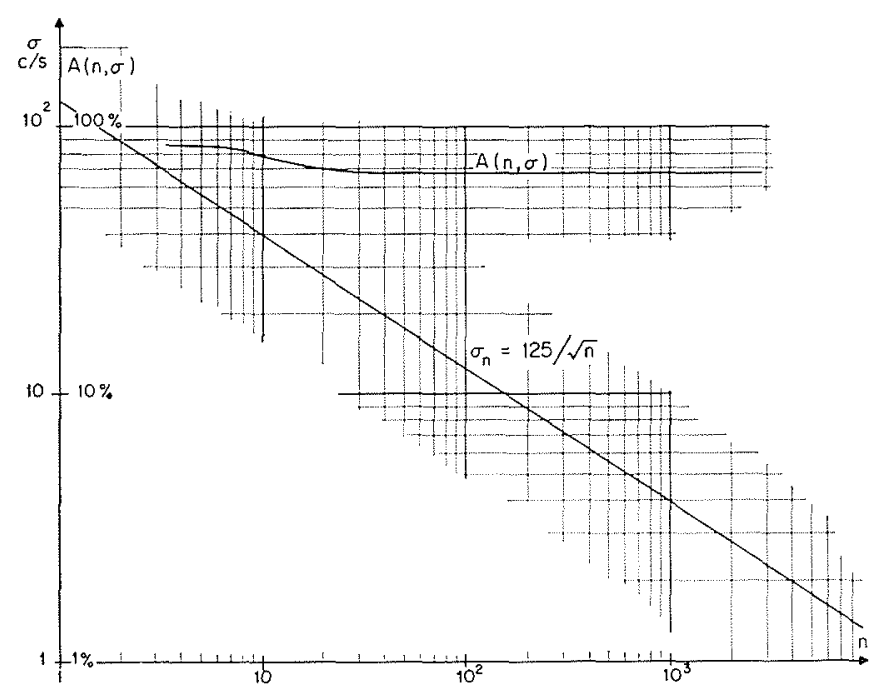

7/ Détection statique. Ecart quadratique, sa probabilité en fonction du nombre de grains.

\subsubsection{Détection Statique et GRanulométrie NON UNi- FORME.}

\section{Généralités.}

Supposons maintenant l'existence de grains de diamètres variables répartis suivant la courbe granulométrique du sédiment étudié.

A moins d'admettre une loi donnée de répartition granulométrique, ce qui ne saurait satisfaire chacun, un tel problème ne peut être traité que sur des cas particuliers. Nous donnons cependant en [1] les équations complètes qui régissent le phénomène.

Nous avons donc choisi l'application à deux cas particuliers: le sable de Loire et celui de Dunkerque.

Nous ne reprendrons pas les résultats complets et nous nous attacherons simplement à déterminer $\sigma_{n}$ qui seul intervient dans le critère de la condition aux limites ci-dessus définie.

\section{Sable de Loire.}

Il s'agit là d'un sable grossier à granulométrie relativement étendue (fig. 9) de diamètre moyen $d_{m}=0,79 \mathrm{~mm}$.

La fonction $\Pi_{1}$ étant déterminée point par point, $\sigma_{1}$ est obtenue au moyen du théorème de Koenig (7).

Note. - Le diamètre moyen $d_{m}$ est le diamètre de la granulométrie uniforme qui donne le même nombre de grains au gramme que la granulométrie réelle.

Pour le sable étudié, on trouve:

$$
\sigma_{1}=206 \mathrm{cps} \text { et par conséquence } \sigma_{n}=206 / \sqrt{n}
$$

Par conséquent, à même nombre de grains, par rapport à la granulométrie uniforme, l'écart $\sigma_{n}$ est considérablement augmenté.

En considérant le même critère que précédemment $\sigma_{n}=15$, on trouve $n=190$ grains et :

$$
\mathrm{P} \mathrm{kg} / \mathrm{Ci}=236 d_{m}{ }^{3}=128 \mathrm{~kg} / \mathrm{Ci} \mathrm{d}{ }^{102} \mathrm{Tr}
$$

\section{Sable de Dunkerque.}

Nous sommes ici dans le cas d'un sable fin à granulométrie relativement peu étendue, de diamètre moyen $d_{m}=0,17 \mathrm{~mm}$ (fig. 10 ).

L'application du processus conduit à :

et :

$$
\sigma_{n}=154 / \sqrt{n} \text { qui donne } n=106
$$

$$
\mathrm{Pkg} / \mathrm{Ci}=147 d_{m}{ }^{3}=7,2 \mathrm{~kg} / \mathrm{Ci} \mathrm{d}^{192} \mathrm{Ir}
$$

\section{Généralisation.}

Si pour les trois granulométries étudiées (uniforme, sable de Loire, sable de Dunkerque), nous portons sur un même graphique, les points de coordonnées :

$$
\sigma_{1}, \mathrm{~L}(\mathrm{~h} / 2)
$$

$\mathrm{L}(\mathrm{h} / 2)$ étant la largeur à mi-hauteur de la courbe des fréquences granulométriques (fig. 9).

Ces trois points sont approximativement alignés (fig. 11) sur la droite d'équation:

$$
\sigma_{1}=125+80 \frac{\mathrm{L}(h / 2)}{d_{m}}=125\left[1+\frac{0,64 \mathrm{~L}(h / 2)}{d_{m}}\right]
$$

relation qui permet de calculer $\sigma_{1}$ puis $\sigma_{n}$ pour une granulométrie quelconque.

La masse de traceur à immerger sera donc :

$$
\mathrm{P}_{\left(\mathrm{k} \mathrm{g} / \mathrm{Ci} \mathrm{d}^{\prime 122 \mathrm{Ir})}\right.}=91 d_{m^{3}}\left[1+\frac{0,64 \mathrm{~L}(h / 2)}{d_{m}}\right]
$$




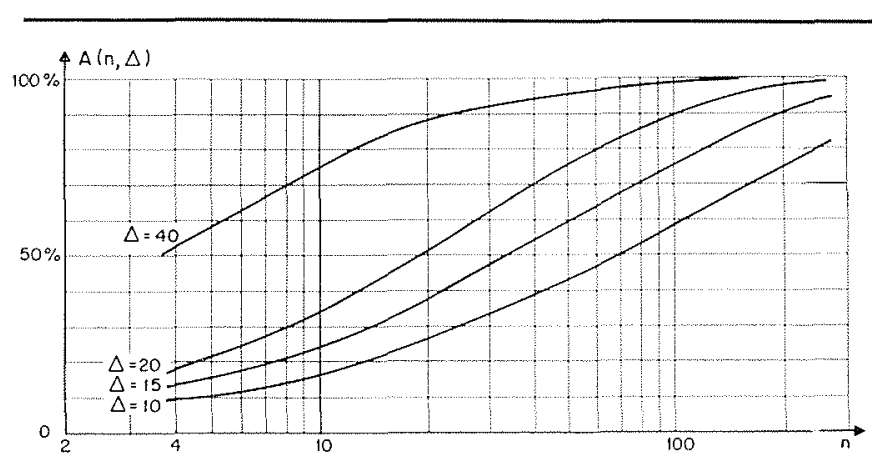

8/

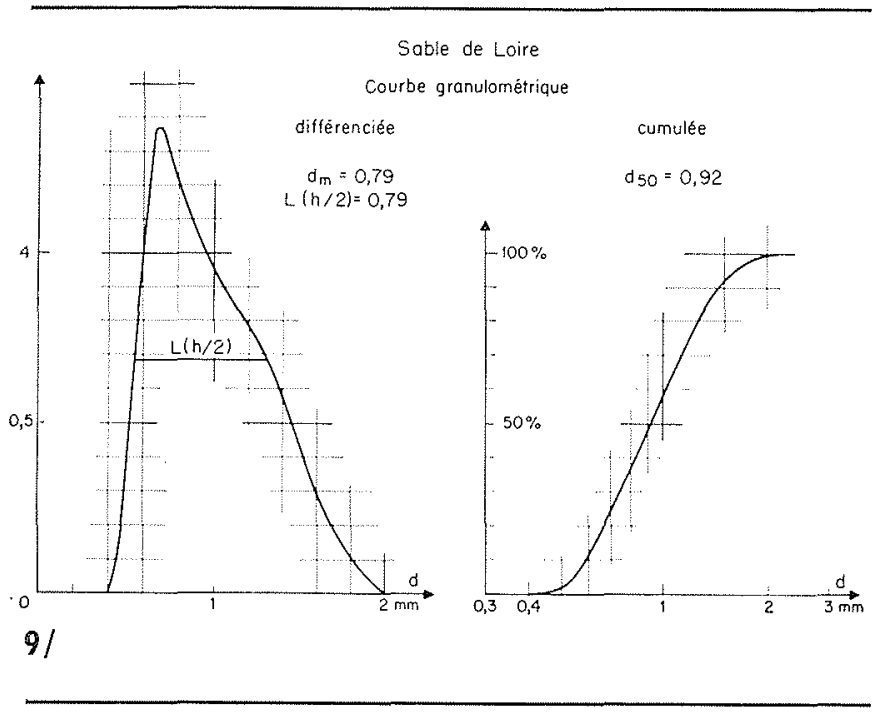

8/ Détection statique. Probabilité associée à un intervalle de comptage donné.

9/ Coube granulométrique. Sable de Loire.

10/ Courbe granulométrique. Sable de Dunkerque.

11/ Détection statique. Détection dynamique. Influence de la granulométrie sur $\sigma_{1}$.

12/ Détection dynamique. Surface d'influence.

Certes nous ne prétendons pas, d'une loi établie sur trois points conclure qu'elle est générale; d'autres exemples devront être calculés, mais l'équation ci-dessus est cependant susceptible de fournir un ordre de grandeur valable.

\subsection{Détection dynamique.}

\subsubsection{Définition et Conditions d'Étude.}

On appelle détection dynamique, toute détection dans laquelle la sonde, montée sur un traineau, est tractée sur le lit à examiner. Ce faisant, la sonde examine une infinité de répartitions de grains, dont la position dans l'espace est fixe, mais varie si on se rapporte aux positions successives du détecteur.

Ce type de détection est quasi général et est celui adopté systématiquement en France.

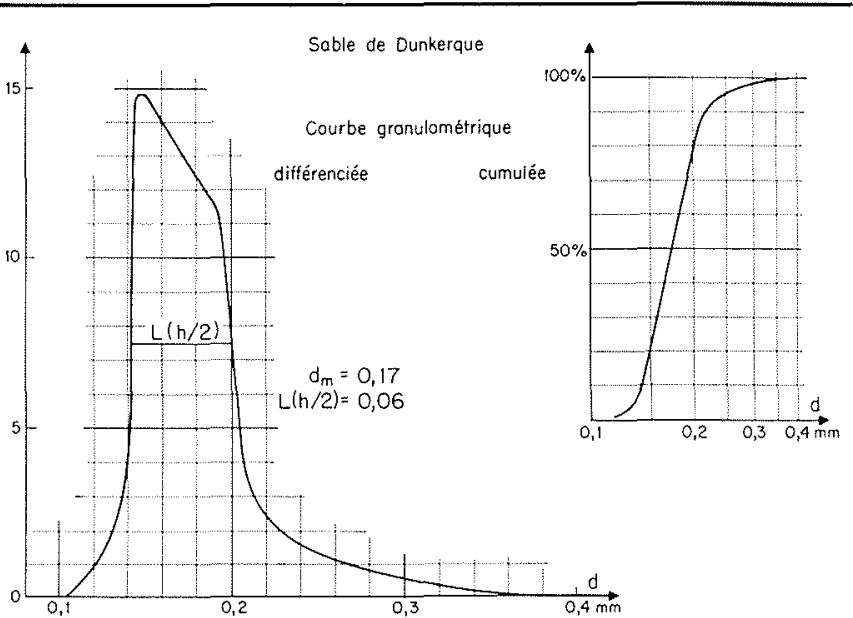

$10 /$

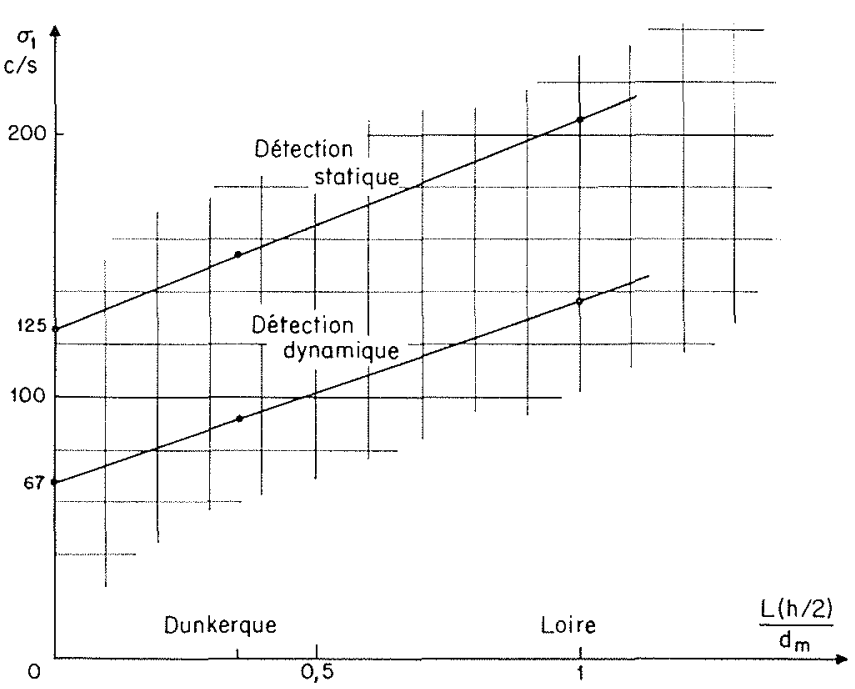

$11 /$

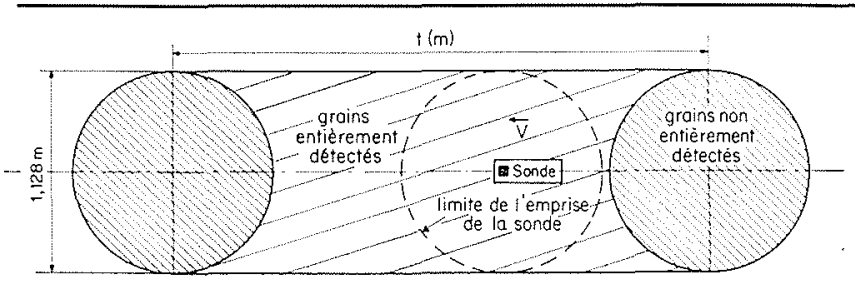

$12 /$

Nous allons examiner ici le cas de la sonde aux caractéristiques définies en 3.1 , détectant un sédiment ensemencé superficiellement de grains radioactifs avec une activité de $1 \mu \mathrm{Ci} \mathrm{d}^{1192} \mathrm{Ir}$ au $\mathrm{m}^{2}$, en se déplaçant sur ce fond à une vitesse uniforme de $1 \mathrm{~m} / \mathrm{s}$, les informations étant totalisées dans une échelle de comptage et décodées régulièrement toutes les $k$ secondes.

Dans ces hypothèses, la sonde détecte les informations en provenance d'une surface formée d'un rectangle de largeur $1,128 \mathrm{~m}$ (diamètre du cercle de surface $1 \mathrm{~m}^{2}$ ), de longueur $t$ mètres, fermé à ses deux bouts par des demi-cercles. Notons qu'avec une telle disposition, certains grains, situés aux extrémités de cette surface sont détectés incomplètement à chaque séquence de comptage. Nous supposerons, ce qui n'altère pratiquement pas les résultats, que cette zone réelle est équivalente à celle obtenue en considérant uniquement le rectangle mais dans lequel toute entité marquée serait entièrement détectée (fig. 12). 


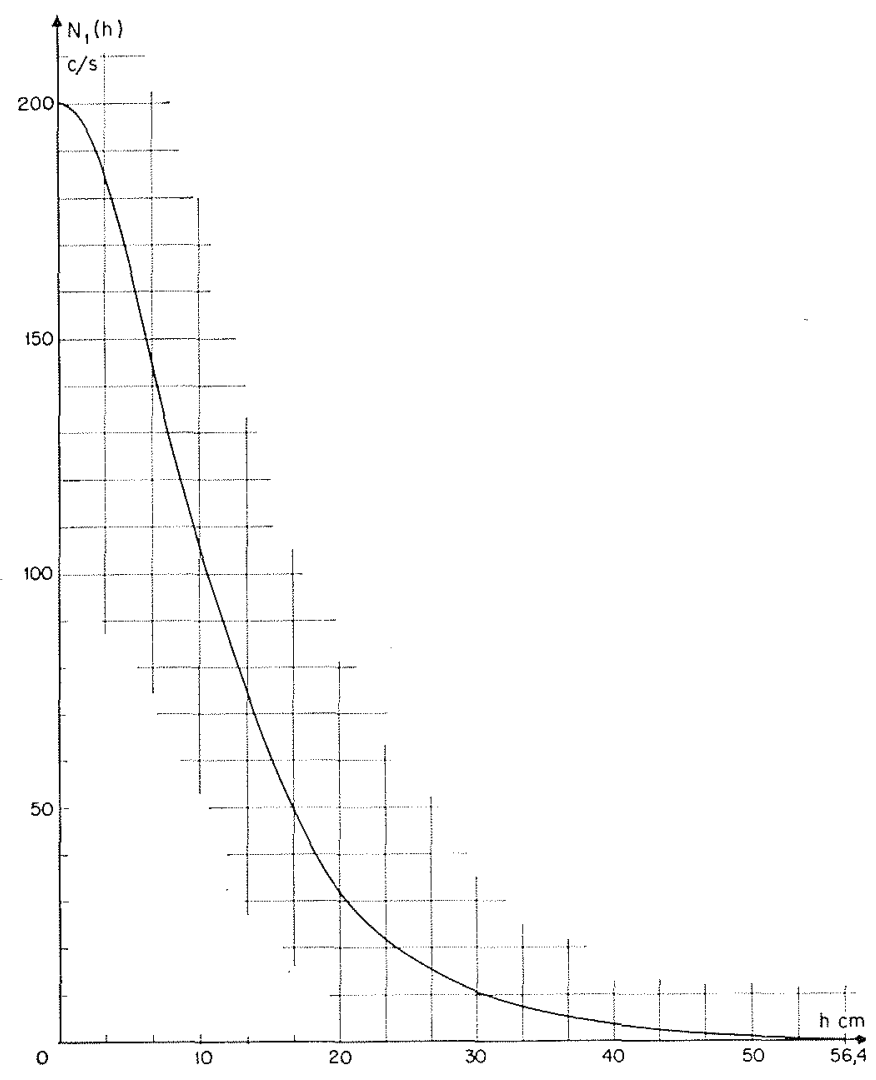

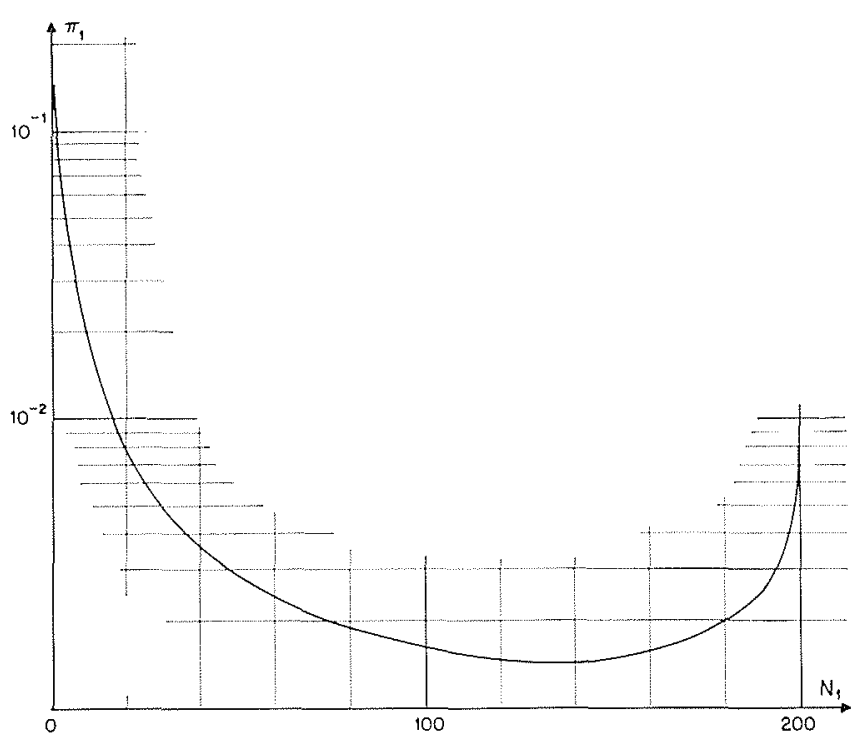

14/ Détection dynamique. Densité de probabilité $\Pi_{1}\left(N_{1}\right)$.

\subsubsection{DÉtection DYNamiQue ET GRANULOMÉTRIE UNIFORME.}

\section{Calculs.}

Pour un grain quelconque $\mathrm{C}$, à un instant donné, la sonde délivre un taux de comptage $\gamma(t)$. La détection de ce grain étant terminée, il a fourni un nombre de chocs totaux :

$$
\mathrm{N}_{1}(h)=\int_{t} \gamma(t) d t
$$

qui ne dépend plus que de sa distance $h$ au trajet de la sonde et qui peut être déterminée à la suite d'un étalonnage en laboratoire (fig. 13).

La probabilité élémentaire qu'un grain soit à une distance $h$ de ce trajet est :

$$
d \mathrm{P}=\frac{d h}{1,128}
$$

La loi de probabilité $\Pi_{1}$ est donc:

$$
\Pi_{1}=\frac{d \mathrm{P}}{d \mathrm{~N}_{1}}=\frac{1}{1,128} \frac{d h}{d \mathrm{~N}_{1}}
$$

$d h / d \mathrm{~N}_{1}$ étant obtenu à partir de la connaissance de $\mathrm{N}_{1}(h)$, $\Pi_{1}\left(N_{1}\right)$ est représenté sur la figure 14 .

A partir de là, le calcul va se dérouler de manière tout à fait analogue à celui mené précédemment.

Les fonctions $\sigma_{1}$ seront déterminées par applications successives de l'équation (3), résolue par le programme mis au point à cette fin.

L'écart quadratique $\sigma_{1}$, trouvé par application du théorème de Koenig servira à déterminer :

$$
\sigma_{n}=\frac{\sigma_{1}}{\sqrt{n}}
$$

Les probabilités associées $\mathrm{A}(n, \sigma)$ à $\sigma_{n}$ et celles associées à des intervalles $\pm \Delta, A(n, \Delta)$ autour de $\bar{N}$ seront déterminées graphiquement.

Les résultats trouvés font l'objet des figures $15,16,17$, 18.

Fig. 15. - Fonctions de probabilité pour $n=8,16$ et 32 grains;

Fig. 16. - $\mathrm{N}_{p}$ et $\mathrm{N}_{p} / \overline{\mathrm{N}}=f(n)$ relatifs aux taux de comptage le plus probable;

Fig. 17. - L'écart quadratique $\sigma_{n}$ et sa probabilité associée $\mathrm{A}(n, \sigma)$ :

$$
\sigma_{n}=67,5 / \sqrt{n}
$$

Fig. 18. - Les pourcentages de cas possibles appartenant à un intervalle donné $\mathrm{N} \pm \Delta: \mathrm{A}(n, \sigma)$.

Note importante. - $n$ n'est plus ici le nombre de grains par $\mathrm{m}^{2}$ mais celui vu par la sonde dans le domaine de comptage. Ce dernier a pour surface 1,128 v.t $t=1,128 \mathrm{k}$. Le nombre moyen de grains par $\mathrm{m}^{2}$ est:

$$
n / 1,128 k
$$

transportant une activité de $1 \mu \mathrm{Ci}$.

Masse minimale de sédiment marqué à immerger.

Pour le même critère que précédemment à savoir un écart quadratique relatif de $30 \%$ correspondant à une déviation standard de $15 \mathrm{cps}$, on a :

$$
\sigma_{n}=15=67,5 / \sqrt{n}
$$

qui donne $n=20$ grains.

Pour ces 20 grains, la probabilité associée est de $68 \%$ (fig. 17). 


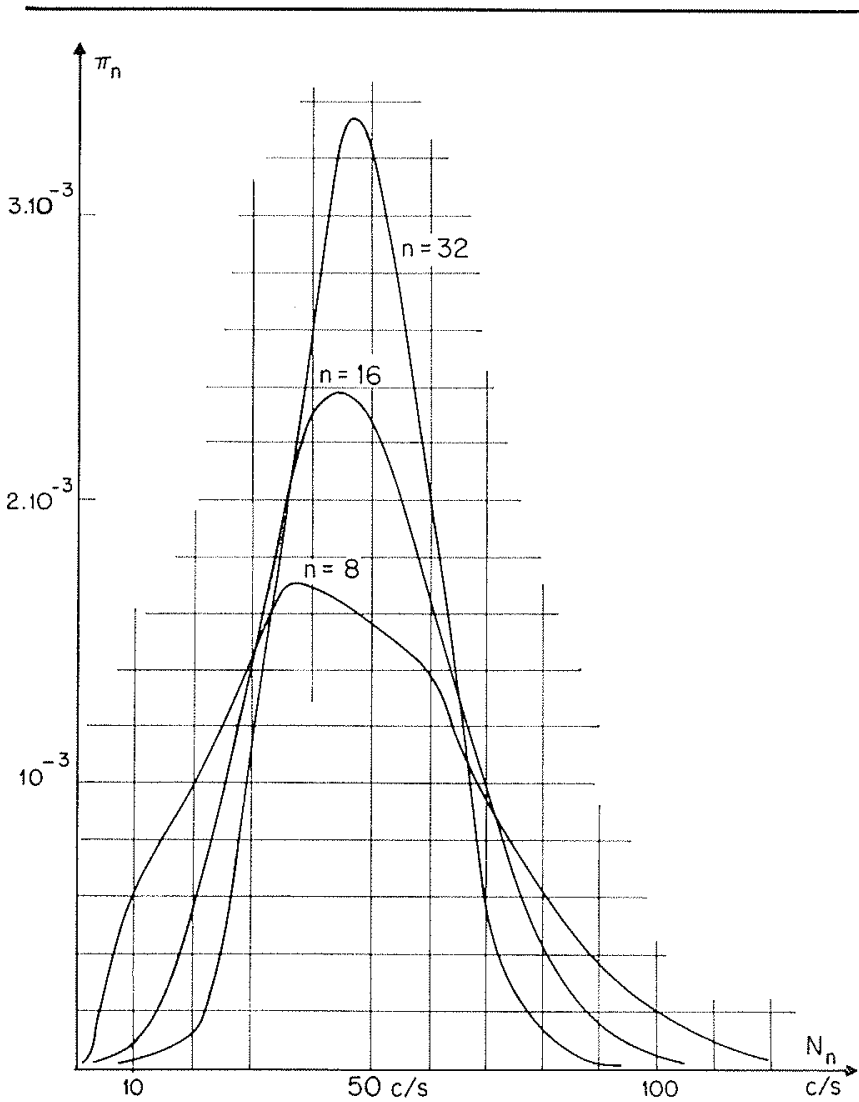

$15 /$ Détection dynamique. Densité de probabilité $\Pi_{5}, \Pi_{16}, \Pi_{3: t}$

Il faut donc 20 grains, sur $1,128 \mathrm{k \mu Ci} \mathrm{d}{ }^{192} \mathrm{Ir}$, correspondant à $\mathrm{P}$ kilogramme par $\mathrm{Ci}$ :

$$
\mathrm{P} \mathrm{kg} / \mathrm{Ci}=(24,8 / k) d^{3}
$$

$d$ en $\mathrm{mm}$.

Ainsi pour un diamètre uniforme de $1 \mathrm{~mm}$ et une séquence de comptage de $3 \mathrm{~s}$ et une vitesse d'exploration de $1 \mathrm{~m} / \mathrm{s}$ :

$$
\mathrm{P} \mathrm{kg} / \mathrm{Ci}=8,3 \mathrm{~kg} / \mathrm{Ci} \mathrm{d}^{192} \mathrm{Ir}
$$

Soit 11 fois moins qu'en détection statique.

\subsubsection{DÉtection DYNAMIQUe ET GRANULOMÉtRIE NON UNI- FORME.}

Comme en détection dynamique, seul l'écart quadratique sera calculé avec applications aux sables de Loire et de Dunkerque.

On trouve :

Sable de Loire : $d_{m}=0,79 \mathrm{~mm}$.

$$
\sigma_{1}=138 \mathrm{cps} \quad \sigma_{n}=138 / \sqrt{n}
$$

qui donne pour $\sigma_{n}=15, n=84$ grains.

d'où :

$$
\mathrm{P} \mathrm{kg} / \mathrm{Ci}=\frac{92 d_{m}{ }^{3}}{k}=\frac{45}{k} \mathrm{~kg} / \mathrm{Ci} \text { d'iridium }
$$

Soit $15,3 \mathrm{~kg}$ par Curie d"192 Ir pour un temps de comptage de $3 \mathrm{~s}$ ( 8 fois moins qu'en détection statique), et une vitesse d'exploration de $1 \mathrm{~m} / \mathrm{s}$.

Sable de Dunkerque $d_{m}=0,17 \mathrm{~mm}$.

$$
\sigma_{1}=92 \mathrm{cps} \quad \sigma_{n}=92 / \sqrt{n}
$$

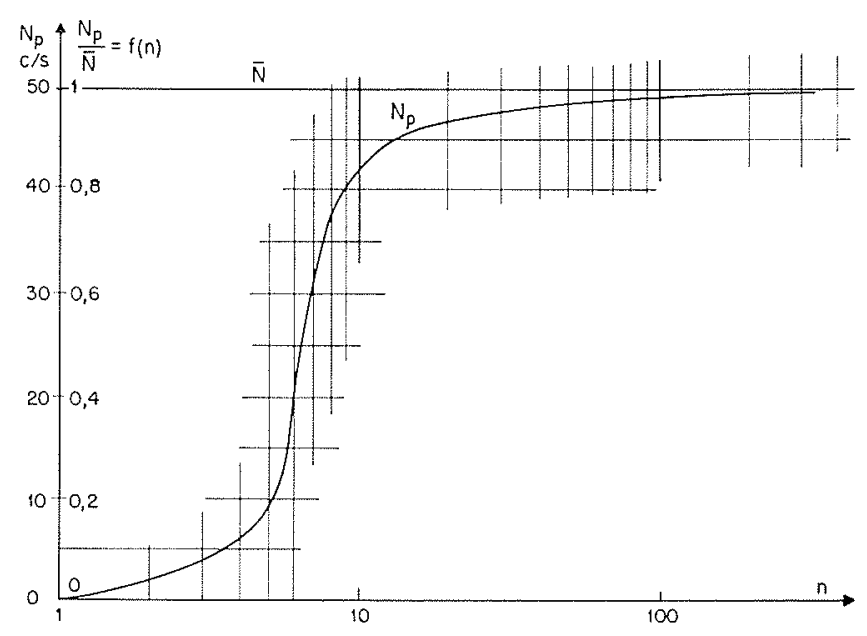

16/ Détection dynamique. $\mathrm{N}_{p}$ et $\mathrm{N}_{z} / \mathrm{N}$ en fonction du nombre de grains.

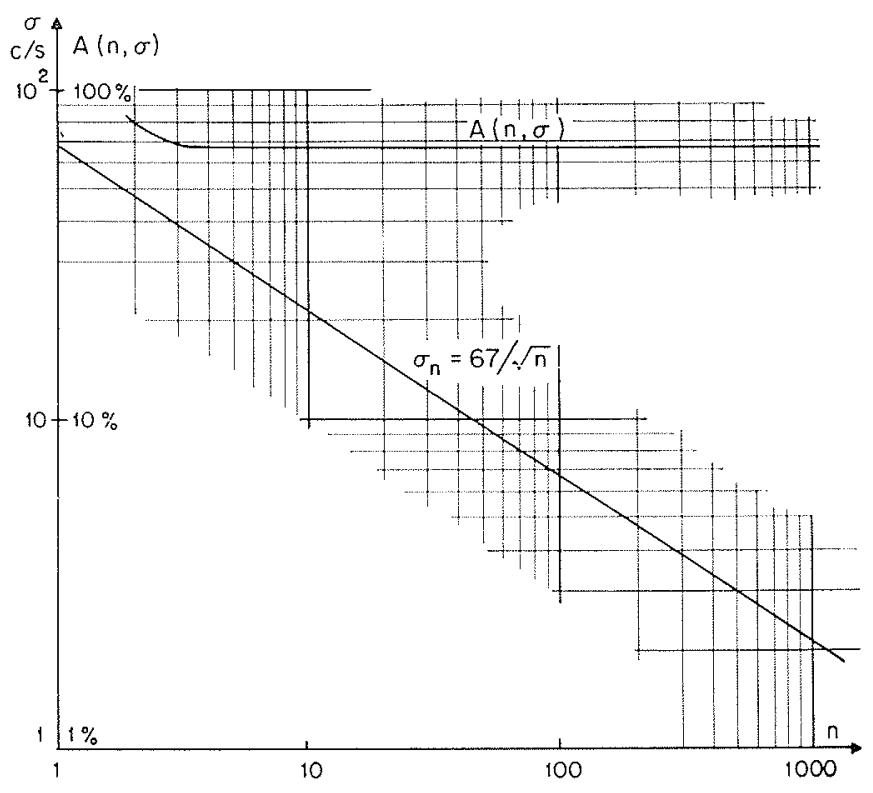

17/ Détection dynamique. Ecart quadratique, sa probabilité en fonction du nombre de grains.

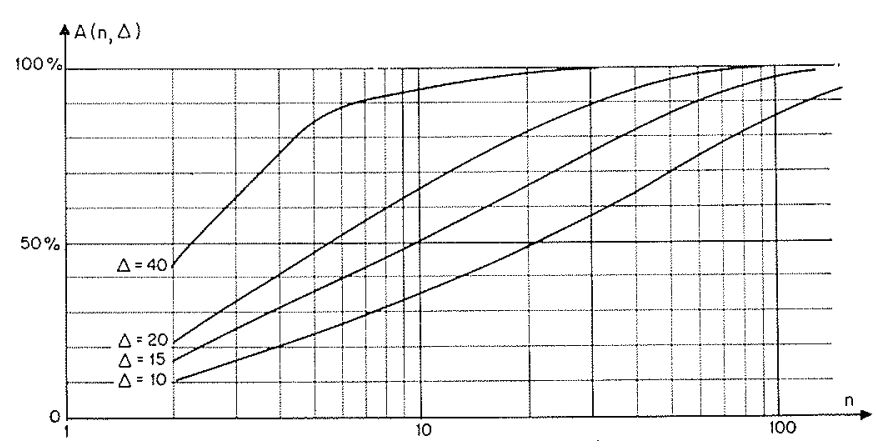

18/ Détection dynamique. Probabilité associée à un intervalle de comptage donné. 
qui donne pour $\sigma_{n}=15, n=37$ grains.

d'où :

$$
\mathrm{P} \mathrm{kg} / \mathrm{Ci}=\frac{40,5 d_{m}{ }^{3}}{k}=\frac{2,0}{k} \mathrm{~kg} / \mathrm{Ci} \text { d'iridium }
$$

Soit environ $0,670 \mathrm{~kg}$ par curie d'192 Ir pour un temps de comptage de $3 \mathrm{~s}$ (11 fois moins qu'en détection statique).

\section{GÉNÉRALISATION :}

De la même manière que précédemment, les trois points de coordonnées :

$$
\sigma_{1}, \frac{\mathrm{L}(h / 2)}{d_{m}}
$$

sont alignés sur la droite d'équation (fig, 11):

$$
\sigma_{1}=67,5+70 \frac{\mathrm{L}(h / 2)}{d_{m}}=67,5\left[1+1,05 \frac{\mathrm{L}(h / 2)}{d_{m}}\right]
$$

qui conduit à la généralisation :

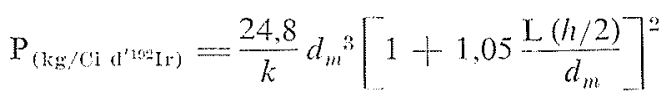

Le terme entre parenthèses traduisant l'effet de la granulométrie avec les réserves posées au paragraphe analogue en détection statique.

\subsection{Conclusions.}

Ainsi donc, les procédés de calcul exposés au paragraphe II ont permis de mener à bien la détermination des caractéristiques des lois de distribution et sur un critère, qui certes est discutable, établir des relations générales définissant les masses minimales à immerger. Ces formules sont par ailleurs immédiatement adaptables à un autre critère.

Les calculs ont été menés tant pour une détection statique que pour une détection dynamique et ils ont montré que les masses à immerger étaient environ dix fois plus petites pour la détection dynamique: La détection statique coûte cher en masse de sédiment marqué à immerger.

Rappelons cependant que ces formules ne sont valables que pour les seules conditions décrites en 3.1 , en particu-

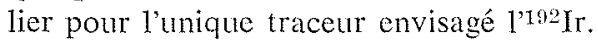

Nous allons maintenant tenter de généraliser les calculs faits et envisager l'influence de différents facteurs. L'étude de l'influence exacte de chacun d'eux ne peut être menée à bien complètement et surtout elle ne peut être rigoureuse. Mais nous estimons que les informations qui vont être fournies sont suffisamment précises pour représenter un ordre de grandeur satisfaisant de la réalité.

\section{4. - Générclisotions Influence de divers facteurs}

\subsection{Présentation générale du problème.}

D'une façon tout à fait générale, le problème peut être exposé de la manière suivante:

Avec un ensemble de détection, quels que soient sa composition type et le nombre de détecteurs, on explore les limites d'une tâche radioactive d'un émetteur donné. Dans ces zones, on considère que l'ensemble utilisé fournit un signal net ou sensibilité de $\varphi$ cps pour une activité de $1 \mu C i$ répartie dans le volume de section droite $1 \mathrm{~m}^{2}$ et de hauteur: l'épaisseur de transport. L'ensemble de détection détecte pendant la mesure une surface de $S \mathrm{~m}^{2}$ contenant une activité $S \mu C i$.

Compte tenu des prospections préalables du bruit de fond on estime que l'on considérera comme significatif tout taux de comptage net de $\mathrm{R}_{f}$ cps; on désire simultanément que la déviation standard due aux seules fuctuations statistiques du nombre de grains sous le détecteur soit inférieure ou égale à une valeur que l'on se fixe : $\sigma_{\mathrm{R}}$.

Connaissant la sensibilité $\varphi$ de l'ensemble de détection, le taux de comptage limite $\mathrm{R}_{i}$ et la déviation standard associée $\sigma_{\mathrm{R}}$, quelle est la masse minimale de traceur qu'il faut immerger?

Rappelons que dans le chapitre 3 précédent, voir (paragraphe 3.1) les calculs sont faits pour une sensibilité $\varphi=f_{0}$ (activité en surface et $\mathrm{S}=1 \mathrm{~m}^{2}$ ) pour un taux limite $\mathrm{R}_{c}=50 \mathrm{cps}$ et pour une déviation standard relative :

$$
\sigma_{\mathrm{R}} / \mathrm{R}_{c}=30 \%
$$

(fin du paragraphe 3.2.2).

Pour résoudre le problème, supposons connue la déviation standard $\sigma$ de la distribution $\Pi$ obtenue dans les conditions réelles de détection pour un grain unique d'activité $\mathrm{S} \mu \mathrm{Ci}$ de l'émetteur choisi; pour $n$ grains d'activité totale $\mathrm{S} u \mathrm{Ci}$ on a:

$$
\sigma_{n}=\sigma / \sqrt{n}
$$

Dans ces conditions on a une déviation $\sigma_{n}$ pour un taux de comptage $\varphi$ (et une activité $S \mu C i$ ) et on veut une déviation $\sigma_{\mathrm{I}}$ pour un taux de comptage $\mathrm{R}_{o}$ et une activité $a$.

On a donc:

$$
\frac{\sigma_{\mathrm{Ii}}}{\sigma_{n}}=\frac{\mathrm{R}_{e}}{\varphi}=\frac{a}{\mathrm{~S}}
$$

d'où :

$$
\frac{\sigma_{\mathrm{I}}}{\sigma} \sqrt{n}=\frac{\mathrm{R}_{\mathrm{e}}}{\varphi}=\frac{a}{\mathrm{~S}}
$$

l'activité est :

$$
a=\mathrm{S} \mathrm{R}_{c} / \varphi
$$

et le nombre de grains présents sous le détecteur devra être :

$$
n=\left(\frac{\sigma}{\sigma_{1:}} \frac{\mathrm{R}_{i}}{\varphi}\right)^{2}
$$

Le nombre de grains par curie sera donc $\mathrm{K}$ :

$$
\mathrm{K}=\frac{10^{6} n}{a}=\frac{\mathrm{R}_{e}}{\rho}\left(\frac{\sigma}{\sigma_{\mathrm{R}}}\right)^{2} \frac{10^{6}}{\mathrm{~S}}
$$

Pour une granulométrie uniforme, on a $0,720.10^{6} d^{-3}$ grains de diamètre $d$ par kilogranme de sable.

Le poids de ces $\mathrm{K}$ grains ou poids minimal à immerger est :

$$
\mathrm{P}=1,39 \frac{d^{3}}{\mathrm{~S}} \frac{\mathrm{R}_{c}}{\varphi}\left(\frac{\sigma}{\sigma_{\mathrm{R}}}\right)^{2} \mathrm{~kg} / \text { curie }
$$

Ainsi la connaissance simultanée de la sensibilité de la sonde $\varphi$, de l'écart $\sigma$, et le choix a priori des conditions aux limites $\mathrm{R}_{c}$ et $\sigma_{\mathrm{R}}$ permet de déterminer le poids à immerger par Curie de l'émetteur retenu.

\subsubsection{INFLUENCE DES COMPOSANTS ET DE LA GÉOMÉTRIE DU SYSTÈME DE DÉTECTION.}

Le système de détection peut être composé d'un ou de plusieurs détecteurs placés dans des positions quelconques. 
On supposera dans ce qui suit que la loi de réponse géométrique de cet ensemble est connue et qu'elle est de la forme :

$$
f=f_{0} e^{-\alpha z}
$$

$f$ est le nombre de chocs nets par seconde, délivré par l'ensemble de détection, pour une activité uniforme de $1 \mu \mathrm{Ci} / \mathrm{m}^{2}$ enfouie à la profondeur $z$.

$f_{0}$ et $\alpha$ sont donc des caractéristiques de l'ensemble détecteur, obtenues par étalonnage en laboratoire. Dans la situation actuelle de la méthode, même pour des problèmes à interprétation qualitative, la connaissance de $f_{0}$ et de $\alpha$ nous paraît absolument indispensable, et l'étalonnage en laboratoire de l'ensemble détecteur, réalisé dans les mêmes conditions d'utilisation que sur le terrain (même géométrie avec sondes en place dans leur traîneau, mêmes conditions électriques notamment de seuil de discrimination, etc.) est une opération tout à fait nécessaire.

\subsubsection{La déviatron STANDARD.}

Rappelons que dans la formule générale (15) $\sigma$ est la déviation standard associée à $I I$ : distribution des probabilités d'obtention d'un taux de comptage fourni par un grain unique d'activité $\mathrm{S} \mu \mathrm{Ci}$ pour toutes les positions de celui-ci dans le volume vu par le détecteur, volume de surface apparente $\mathrm{S} \mathrm{m}^{2}$. Nous supposerons que cet écart est indépendant de la loi de réponse géométrique de l'ensemble de détection. Ainsi $\sigma$ reste fonction de l'enfouissement du traceur, du mode de détection utilisé (statique ou dynamique) et bien entendu de la nature de l'émetteur choisi.

\subsection{Efiet d'eníouissement. Valeur de $\sigma$. Valeur de $\varphi$.}

Supposons qu'aux limites, l'expérimentateur puisse supposer un enfouissement sur une épaisseur $\mathrm{E}$, avec une loi de répartition du traceur en profondeur $c(z)$.

Comme nous ne recherchons qu'un ordre de grandeur, nous supposerons que le traceur est uniformément réparti en profondeur :

Calcul de $\sigma$.

$$
c(z)=\mathrm{Cte}
$$

Chaque couche élémentaire contient un même nombre de grains d'activité totale $d \mathrm{~A}=a d z$ donnant un signal net :

$$
d r=(a / S) f_{0} e^{-\alpha z} d z
$$

La distribution des probabilités des taux de comptage correspondant à cette couche: $\Pi(z)$ a une déviation standard $\sigma(z)$ pour un nombre de grains $n$, on a:

$$
\sigma(z)=\sigma_{n} / \sqrt{n}
$$

$\sigma_{\mu}$ est l'écart pour un grain unique d'activité $a$ situé à la profondeur, on peut le relier, à l'écart $\sigma_{1}$ correspondant à un grain unique d'activité $1 \mu \mathrm{Ci}$ situé en surface et calculé dans les parties 2 et 3 , on a:

$$
\sigma_{z}=\sigma_{u} / \sqrt{n}=(a / S) \sigma_{1} e^{-\alpha z} / \sqrt{n}
$$

D'autre part, l'écart fourni par la somme de toutes les couches élémentaires précédentes est donné par:

$$
\begin{gathered}
\Sigma^{2}=\int \sigma^{2}(z) d z \\
\Sigma^{2}=\frac{a^{2} \sigma_{1}^{2}}{\mathrm{~S}^{2} n} \int_{0}^{\mathrm{D}} e^{-2 \alpha z} d z \\
\Sigma^{2}=\frac{a^{2} \sigma_{1}^{2}}{\mathrm{~S}^{2} n}\left(\frac{1-e^{-2 \alpha \mathrm{E}}}{2 \alpha}\right)
\end{gathered}
$$

Ceci est l'écart pour $n$ grains par couche, soit $n \mathrm{E}$ grains au total.

On a donc pour un grain dans le volume détecté :

$$
\begin{gathered}
\sigma=\Sigma \sqrt{n \mathrm{E}} \\
\sigma^{2}=\frac{a^{2} \sigma_{1}^{2}}{\mathrm{~S}^{2}} \frac{1-e^{-2(\mathrm{E}}}{2 \alpha} \mathrm{E}
\end{gathered}
$$

Calcul de $\varphi$.

D'autre part la somme des taux de comptage est :

$$
\mathrm{R}=-\int r d z=\frac{a}{\mathrm{~S}} f_{0} \frac{1-e^{-a \mathrm{~F}}}{\alpha}
$$

L'activité totale est $\mathrm{A}=a \mathrm{E}$ donc :

$$
\mathrm{R}=\frac{\mathrm{A}}{\mathrm{SE}} f_{0} \frac{1-e^{-\left(\mathrm{YP}_{2}\right.}}{\alpha}
$$

Si l'activité A vaut $\mathrm{S} \mu \mathrm{Ci}, a=\mathrm{S} / \mathrm{E}$ et $\mathrm{R}$ vaut $\varphi$ :

Calcul de $P$.

$$
\varphi=f_{0} \frac{1-e^{-\alpha \mathrm{E}}}{\alpha \mathrm{E}}
$$

D'où en reportant $\varphi$ et $\sigma^{2}$ dans (15):

$$
\mathrm{P}=1,39 \frac{d^{3}}{\mathrm{~s}} \frac{\mathrm{R}, \sigma_{1}^{2}\left(1-e^{2} \mathrm{E}^{2}\right)}{f_{0} \sigma_{\mathrm{R}^{2}} 2\left(1-e^{-\alpha \mathrm{E}}\right)}
$$

\section{REMARQUES IMPORTANTES.}

$1^{\circ}$ Pour une épaisseur nulle, on trouve bien $\varphi=f_{0}$.

$2^{\circ}$ Le poids $P$ est maximum pour la valeur $\mathrm{E}=0$ et vaut alors le double de la valeur minimale obtenue pour $\mathrm{E}=\infty$. Il semble donc que le calcul précis fait dans les parties 2 et 3 soit bien un calcul fait dans le sens de la sécurité.

$3^{\circ}$ II ne faut pas oublier que dans cette formule $\sigma_{1}$ est relatif à un grain d'activité $1 \mathrm{uCi}$ en surface.

\subsection{Emploi d'autres traceurs. Facteur $\sigma_{1}$}

Nous allons voir l'emploi d'autres traceurs dans le cadre de la formule (16), c'est-à-dire que nous allons donner les valeurs $\sigma_{1}$ pour d'autres traceurs.

Outre l'iridium 192 nous avons retenu le chrome 51, le césium 137, le tantale 182.

Certes, le césium 137 n'est pas un traceur utilisable en dynamique des sédiments mais il a été choisi pour ses caractéristiques radioactives : énergie et nombre de photons émis par désintégration.

Pour une détection dynamique nous avons trouvé :

\begin{tabular}{|c|c|}
\hline RADió́LÉMENT & $\sigma_{1}$ \\
\hline & \\
\hline $19 \mathrm{Cr}$ & 3,5 \\
$192 \mathrm{Ir}$ & 67,5 \\
$137 \mathrm{Cs}$ & 32,4 \\
$182 \mathrm{Ta}$ & 62,5 \\
& \\
\hline
\end{tabular}

Exprimons $\sigma_{1}$ en fonction de $\mu_{0}$ coefficient d'atténuation dans l'eau du rayonnement de l'émetteur considéré et $\mathrm{I}_{\gamma}$ la constante d'ionisation de cet émetteur. On trouvera une liste de $I_{\gamma}$ par exemple dans [3]. 


\begin{tabular}{|c|c|c|c|}
\hline RADIOÉLÉMENT & $\mu_{v}$ en $\mathrm{cm}^{-1}$ & $\begin{array}{cccc}\mathrm{I}_{\gamma} & \text { en } & \mathrm{mR} / \mathrm{h} \\
\text { par } & \mathrm{Ci} & \grave{a} & 1 \mathrm{~m}\end{array}$ & $\mu_{0}{ }^{l} \gamma$ \\
\hline${ }^{51} \mathrm{Cr}$ & 0,117 & 21 & 2,46 \\
\hline $192 \mathrm{Yr}$ & 0,108 & 500 & 54,0 \\
\hline $137 \mathrm{Cs}$ & 0,086 & 300 & 25,8 \\
\hline${ }^{182} \mathrm{Ta}$ & 0,075 & 590 & 44,3 \\
\hline
\end{tabular}

D'après la figure 19 , on voit que $\sigma_{1}$ peut être considéré, à $6 \%$ près, comme une fonction linéaire de $\mu_{0} I_{\gamma}$, d'équation :

$$
\sigma_{1}=1,34 \mu_{0} \mathrm{I}_{\gamma}
$$

De la même manière, en détection statique, on prendra pour $\sigma_{1}$, une relation linéaire:

$$
\sigma_{1}=2,5 \mu_{0} \mathrm{I}_{\gamma}
$$

Les équations 17 et 17 bis permettent de déterminer la masse à immerger quel que soit le traceur choisi par l'une des deux équations :

$$
\mathrm{P}_{a}=2,5 \frac{d^{s}}{\mathrm{~S}} \frac{\mathrm{R}_{c}}{f_{0}} \frac{1-e^{-2 \mathrm{~W}}}{2\left(1-e^{-\alpha \mathrm{R}}\right)}\left(\frac{\mu_{0} \mathrm{I} \gamma}{\sigma_{\mathrm{R}}}\right)^{2}
$$

en détection dynamique,

$$
\mathrm{P}_{\mathrm{S}}=8,7 \frac{d^{3}}{\mathrm{~S}} \frac{\mathrm{R}_{\theta}}{f_{0}} \frac{1-e^{-2 \omega \mathrm{W}}}{2\left(1-e^{-\alpha \mathrm{N}}\right)}\left(\frac{\mu_{0} \mathrm{I} \gamma}{\sigma_{\mathrm{R}}}\right)^{2}
$$

en détection statique,

$P_{d}$ et $P_{s}$ en $\mathrm{kg} / \mathrm{Ci}$.

\subsection{Influence de la surface déteciée. Emploi d'un intégrateur.}

- En détection statique, la surface $\mathrm{S}$ est déterminée en laboratoire en même temps que l'emprise « $l »$ du système de détection.

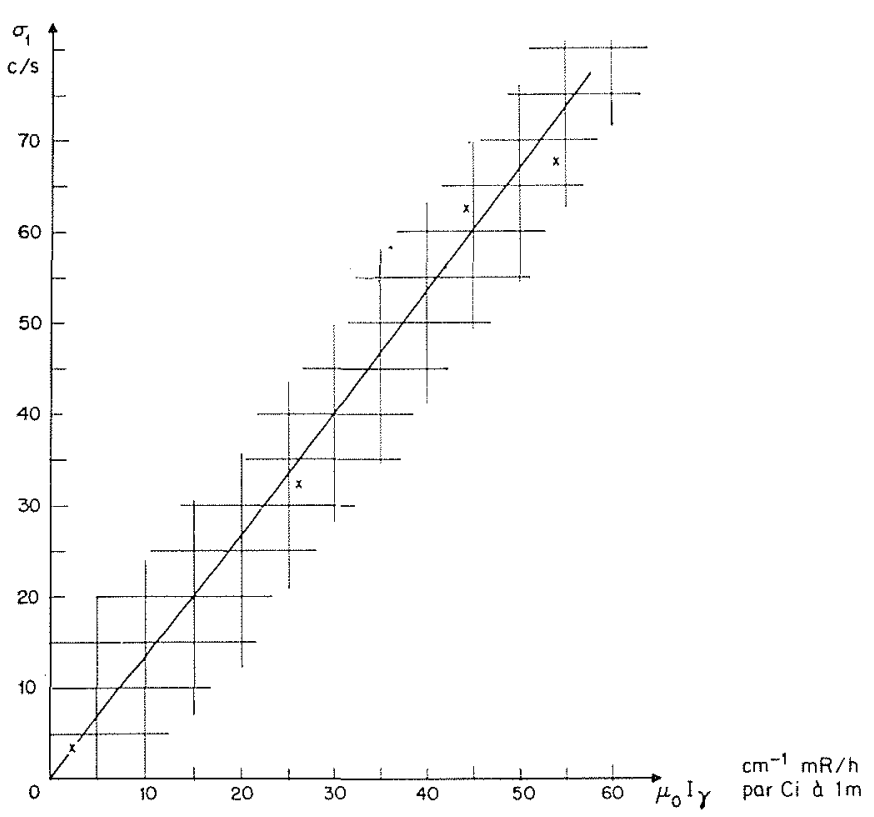

$19 /$ Généralisations à d'autres traceurs $\sigma_{1}$ fonction du produit $\mu_{0} \mathrm{I}_{\gamma}$.
- En détection dynamique, la surface examinée est le produit de l'emprise de l'ensemble de détection par la longueur parcourue par le traineau entre deux décodages successifs $v t=k$.

Notons que $t$ :

- sera pris égal à l'intervalle entre deux décodages successifs pour un système utilisant une échelle $t=k$;

- sera pris égal à deux fois la constante de temps $\tau$ de l'appareillage pour l'emploi d'un ictomètre $t=2 \tau$; eu égard à ce que la déviation standard d'un taux de comptage en intégrateur est le même que celui donné par une échelle de comptage pendant deux constantes de temps:

$$
\begin{gathered}
\frac{\sigma_{n}}{n}=\frac{1}{\sqrt{2 \tau n}}=\frac{1}{\sqrt{m}} \\
t=2 \tau
\end{gathered}
$$

On voit ainsi que la masse de traceur nécessaire sera d'autant plus faible que la vitesse de détection sera grande.

\subsection{Effet de la granulométrie.}

Nous retiendrons comme effet de la granulométrie, les deux facteurs précédents déterminés à savoir :

$$
\left(1+1,05 \frac{\mathrm{L}(h / 2)}{d_{m}}\right)=
$$

en détection dynamique,

$$
\left(1+0,64 \frac{\mathrm{L}(h / 2)}{d_{m}}\right)^{2}
$$

en détection statique.

\subsection{Formules générales.}

Compte tenu des généralisations développées aux paragraphes précédents les formules générales des masses à immerger sont :

Détection dynamique.

$\mathrm{P}_{d}=1,25 \frac{d^{3} m}{l v t} \frac{\mathrm{R}_{t}}{f_{0}} \frac{1-e^{-2 \alpha \mathrm{E}}}{1-e^{-\alpha \mathrm{E}}}\left(\frac{\mu_{0} \mathrm{I} Y}{\sigma_{\mathrm{R}}}\right)^{2}\left(1+1,05 \frac{\mathrm{L}(h / 2)}{d_{m}}\right)^{2}$

$\mathrm{P}_{d}$ en $\mathrm{kg} / \mathrm{Ci}$.

avec $t=k$ en détection avec une échelle et $t=2 \tau$ en détection avec un ictomètre.

Détection statique.

$\mathrm{P}_{\mathrm{S}}=4,35 \frac{d^{3}{ }_{m}}{\mathrm{~S}} \frac{\mathrm{R}_{c}}{f_{0}} \frac{1-e^{-2 \alpha \mathrm{F}}}{1-e^{-\alpha \mathrm{T}}}\left(\frac{\mu_{0} \mathrm{I} \gamma}{\sigma_{\mathrm{R}}}\right)^{2}\left(1+0,64 \frac{\mathrm{L}(h / 2)}{d_{m}}\right)^{2}$

$P_{\mathrm{S}}$ en $\mathrm{kg} / \mathrm{Ci}$.

\subsection{Exemple de détermination de la masse minimum à immerger.}

Un expérimentateur envisage une immersion de quatre curies d'198 Au sur un lieu où le sable assez grossier a un diamètre moyen de $0,5 \mathrm{~mm}$ avec une résolution:

$$
\mathrm{L}(h / 2)=0,2 \mathrm{~mm}
$$

$$
\mathrm{S}=l v t
$$

\section{$C$ \\ $h$ \\ " \\ $t$ \\ to \\ at \\ rie \\ dé \\ lar}

qu

vo
ser
un
re

dé

:or

ale

\section{i}

ne

u 


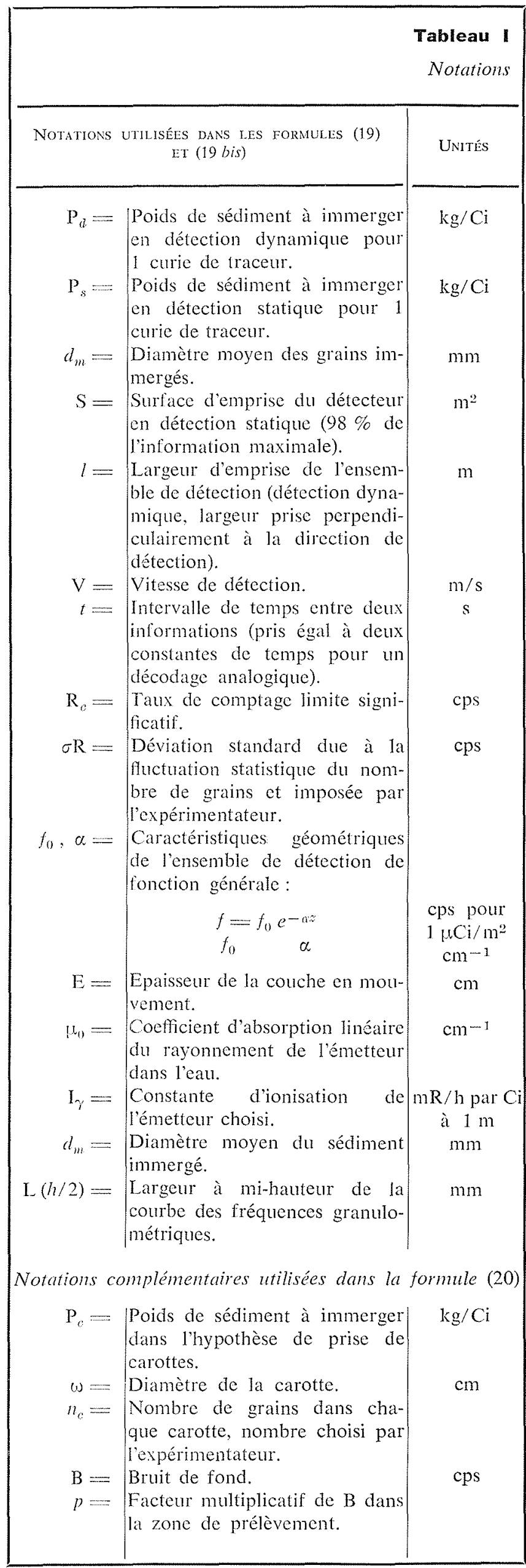

Son ensemble de détection constitué de deux détecteurs à scintillation a une surface d'exploration $\mathrm{S}$ de $1,4 \mathrm{~m}^{2}$ pour une emprise $l$ de $1,25 \mathrm{~m}$ et sa fonction de réponse à ${ }^{1}{ }^{198} \mathrm{Au}$ est :

$$
f=60 e^{-0,150 \%}
$$

Il envisage une détection en dynamique sur un bateau de vitesse moyenne $1,80 \mathrm{~m} / \mathrm{s}$, avec un ictomètre de constante de temps $\tau=1,2 \mathrm{~s}$. Le bruit de fond moyen relevé $\mathrm{B}$ étant de $30 \mathrm{cps}$, l'expérimentateur considère comme significatif tout signal double du bruit de fond, entrainant $\mathrm{R}_{c}=30 \mathrm{cps}$ et il désire que la déviation de ce signal dû à l'effet de la statistique du nombre de grain ne dépasse pas $10 \mathrm{cps}$.

Il considère enfin comme probable un enfouissement de $5 \mathrm{~cm}$ sur les bords de la tâche.

Quelle masse minimale de traceur doit-il immerger?

L'énoncé du problème contient tous les éléments de résolution de (19) excepté $\mu_{0}$ et $I_{\gamma}$. Nous prendrons pour l'198 Au lénergie $410 \mathrm{KeV}$ :

$$
\begin{gathered}
\mu_{0}=0,104 \mathrm{~cm}^{-1} \\
\mathrm{I}_{\gamma}=230 \mathrm{mR} / \mathrm{h} / 1 \mathrm{~m} \text { par Curie }
\end{gathered}
$$

Dans ces conditions, lapplication de (19) donne:

$$
\mathrm{P}_{d}=0,250 \mathrm{~kg} / \mathrm{Ci}
$$

soit un poids total à immerger de $1 \mathrm{~kg}$.

A noter, que dans les mêmes conditions, une détection en statique aurait conduit à :

$$
\mathrm{P}_{s}=2,570 \mathrm{~kg} / \mathrm{Ci}
$$

correspondant à un poids total à immerger de $10,3 \mathrm{~kg}$.

Le tableau 1 ci-contre résume les notations et les unités utilisées et permet d'utiliser au mieux (19) et (19 bis).

\section{5. -- Un cors porticulier: la prise de corrottes}

Examinons pour terminer quelle serait la masse minimale à immerger dans le cas ou lon désire prendre des carottes.

Les calculs seront effectués sur les bases suivantes:

Le carottier a un diamètre $\omega \mathrm{cm}$ correspondant à une surface $\Pi \omega \omega^{2} / 4$. L'opérateur opère sur une zone correspondant à $p$ fois le bruit de fond $B$ et désire, dans chaque carotte, $n_{c}$ grains actifs.

On doit donc avoir $n_{e}$ grains actifs dans une section de $\Pi \omega \omega^{2} / 4$ ce qui correspond à un nombre de grains au $\mathrm{m}^{2}$ de :

$$
\frac{4.10^{4} n_{c}}{\pi \omega^{2}}
$$

fournissant un signal net $p \mathrm{~B}$ avec un ensemble détecteur de sensibilité $\varphi$ (même notation qu'au paragraphe 4).

Dans ces conditions:

4.104 $n_{f} / \Pi \omega^{2}$ grains, de poids :

$$
\frac{4.10^{4} n_{0} d_{m}^{3} \times 1.4 .10^{-6}}{\Pi \omega^{2}} \mathrm{~kg}
$$

doivent transporter une activité de:

$$
\frac{p \mathrm{~B} 10^{--6}}{\varphi} \mathrm{Ci}
$$




\section{G. courtois et al.}

Le poids de 1 curie sera donc:

$$
\begin{gathered}
\mathrm{P}_{c}=\frac{4.10^{4} n_{0} d_{m}{ }^{3} \times 1,4.10^{-6}}{\Pi \omega^{2}} \times \frac{\varphi}{p \mathrm{~B}} \times 10^{\mathrm{s}} \\
\mathrm{P}_{0}=1.78 .10^{1 \frac{n_{c} d_{m}{ }^{3} \varphi}{\omega^{2} p \mathrm{~B}}}
\end{gathered}
$$

ou encore, en prenant pour $\varphi$ la fonction, précédemment établie :

$$
\begin{gathered}
\varphi=\frac{f_{0}\left(1-e^{-i \mathrm{~L}}\right)}{\alpha \mathrm{E}} \\
\mathbf{P}_{0}=\frac{1,78.10^{\prime} n_{c} d_{m}^{3} f_{0}\left(1-e^{-r \mathrm{~L}}\right)}{\omega^{2} p \mathrm{~B} \alpha \mathrm{E}}
\end{gathered}
$$

$P_{c}$ en $\mathrm{kg} /$ curie.

Appliquons cette formule au cas évoqué en 4.7 , en supposant un carottier de $10 \mathrm{~cm}$ de diamètre, dans une zone donnant un signal net égal à 10 fois le mouvement propre et en désirant observer la position d'un minimum de 30 grains actifs dans cette carotte. On suppose par contre aux endroits de prélèvements un enfouissement d'une vingtaine de centimètres.

Dans ce cas l'application de (20) donne alors:

$$
\mathrm{P}_{c}=42,3 \mathrm{~kg} / \mathrm{Ci}
$$

correspondant à un poids total à immerger de $170 \mathrm{~kg}$.

Ainsi donc est-il clairement établi que, pour pouvoir prendre des carottes représentatives, les masses à immerger doivent être considérablement plus élevées que pour une détection en dynamique ou même en statique.

La prise de carottes coûte donc très chère en masses à immerger.

\section{6. - Conclusions}

Nous avons donc établi une série de formules (19), (19 bis) et (20), qui, dans des conditions que nous espérons les plus générales possibles, permettent de calculer les masses minimales à immerger dans le cas:

- d'tne détection dynamique (19):

- d'une détection statique (19 bis);

- de la prise de carottes (20).

Retenons que, en première approximation, les masses à immerger en détection statique sont environ 10 fois plus élevées qu'en détection dynamique, et que les prises de carottes nécessitent à leur tour 10 fois plus environ qu'en détection statique.

Le mode de calcul choisi est généralisable à des conditions diverses. Dans le présent texte, l'application des principes exposés a été faite au tracé des réseaux d'isochocs, tracé que nous reconnaissons comme fallacieux et non représentatif [4], mais il est facilement adaptable à tout autre type de représentation.

Par ailleurs, si pour des raisons technologiques la masse de sédiment est imposée, les formules précédentes permettent :

- soit de déterminer la déviation standard due à la fluctuation du nombre de grains pour un type de détection imposé;

- soit au contraire de choisir les conditions de détection (statique, dynamique, vitesse et emprise de la sonde, traceur, mode et temps d'intégration,...) pour obtenir une déviation standard donnée.

Enfin le présent développement est aussi un moyen puissant d'interprétation permettant d'attribuer une probabilité à un transport observé aux extrémités de la tache radioactive et de ne pas ainsi surestimer un transport en attribuant une valeur trop importante à des zones où l'information peut apparaître comme non significative.

\section{Note.}

En détection statique intervient un calcul par ordinateur. Nous l'avons écrit en Fortran IV pour IBM 360. Nous l'avons nommé STAST.

En détection dynamique interviennent deux calculs:

Le premier donne le calcul rapide de $N_{1}(h)$ : STDYPR.

Le deuxième a un rôle analogue au programme STAST, nous l'avons appelé STADYN.

II n'est pas possible de donner ici le texte et l'explication de ces programmes, mais ils sont tenus par les auteurs à la disposition de toute personne intéressée.

\section{7. - Références}

[1] Sauzay (G.), - Méthode du bilan des taux de comptage d'indicateurs radioactifs pour la détermination du débit de charriage des lits sableux. Rapport C.E.A.R., n” 3 431, 1968.

[2] HALD. - Statistical theory with enginecring applications. John Wiley and Sons, New York.

[3] NuClEonics. - Vol 21, n 8, aô̂t 1963.

[4] Courtors (G.). - Dynamique sédimentaire en France au moyen de traceurs (à paraitre dans la Houille Blanche). 